\title{
Double-soft behavior for scalars and gluons from string theory
}

\author{
Paolo Di Vecchia, ${ }^{a, b}$ Raffaele Marotta ${ }^{c}$ and Matin Mojaza ${ }^{b}$ \\ a The Niels Bohr Institute, University of Copenhagen, \\ Blegdamsvej 17, DK-2100 Copenhagen Ø, Denmark \\ ${ }^{b}$ Nordita, KTH Royal Institute of Technology and Stockholm University, \\ Roslagstullsbacken 23, SE-10691 Stockholm, Sweden \\ ${ }^{c}$ Instituto Nazionale di Fisica Nucleare, Sezione di Napoli, \\ Complesso Universitario di Monte S. Angelo ed. 6, via Cintia, 80126, Napoli, Italy \\ E-mail: divecchi@nbi.dk, raffaele.marotta@na.infn.it, \\ mojaza@nordita.org
}

ABSTRACT: We compute the leading double-soft behavior for gluons and for the scalars obtained by dimensional reduction of a higher dimensional pure gauge theory, from the scattering amplitudes of gluons and scalars living in the world-volume of a $\mathrm{D} p$-brane of the bosonic string. In the case of gluons, we compute both the double-soft behavior when the two soft gluons are contiguous as well as when they are not contiguous. From our results, that are valid in string theory, one can easily get the double-soft limit in gauge field theory by sending the string tension to infinity.

KEYwords: Scattering Amplitudes, D-branes, Bosonic Strings

ARXIV EPRINT: 1507.00938 


\section{Contents}

1 Introduction and results 1

2 Double-soft behavior with $n+2$ gluons $\quad 4$

3 Double-soft behavior with 2 scalars and $n$ gluons 11

4 Double-soft behavior with $n+2$ scalars $\quad 11$

5 Conclusions 13

A Computation of various integrals to the leading order in the double-soft limit 13

\section{Introduction and results}

Soft theorems were in the 1950s and 1960s studied for photons [1-9] and gravitons [5, 6, 10, 11], when they were recognized to be important consequences of local gauge invariance. ${ }^{1}$ More recent discussions of the generic subleading behavior of soft gluons and gravitons were given in refs. [13-15]. The very recent interest in the soft behavior of gravitons and gluons has arisen after work by Strominger and collaborators [16-18], showing that the soft-graviton behavior follows from Ward identities of extended Bondi, van der Burg, Metzner and Sachs (BMS) symmetry [19-23], and that tree-level graviton amplitudes in four spacetime dimensions have a universal behavior through second subleading order in the soft-graviton momentum [24]. This has stimulated the study of the subleading soft behavior in amplitudes with gluons and gravitons, which we will briefly summarize.

For gluons in arbitrary number of dimensions soft theorems were obtained in various ways at tree level [25-31], and discussed for quark-gluon amplitudes in QCD in ref. [32]. Poincaré and gauge invariance as well as a condition arising from the distributional nature of scattering amplitudes have been used in ref. [33] to strongly constrain the soft behavior for gluons and gravitons, while in ref. [34] gauge invariance is shown to completely fix the first two leading terms (up to terms $\mathcal{O}\left(q^{0}\right)$ ) in the case of a gluon, and the first three leading terms (up to terms $\mathcal{O}\left(q^{1}\right)$ ) in the case of a graviton, for any number of space-time dimensions ( $q$ being the soft momentum).

Further study of the subleading soft-graviton theorems in arbitrary number of dimensions were performed in refs. [35-41]. Soft theorems were also studied in the ambitwistor string [42, 43], and their connection with effective field theories was discussed in ref. [44]. As discussed in refs. [34, 45-47], one should note that soft gluon and graviton behaviors are in general modified by loop corrections.

Finally, soft gluon and graviton behavior have also been studied in the framework of string theory in refs. [29, 48-50]. In particular, soft theorems for the dilaton were

\footnotetext{
${ }^{1}$ For a discussion of low-energy theorem for photons see chapter 3 of ref. [12].
} 
discussed in refs. [50-54] and for the anti-symmetric tensor in ref. [50], while soft behavior in amplitudes with massive states was discussed in ref. [55].

Double-soft theorems are now receiving increasing interest, and have been studied for scalars and gluons. The interest in the double-soft behavior arises originally from the analysis of the spontaneously symmetry breaking of a group $G$ keeping a subgroup $H$ unbroken. The presence of Nambu-Goldstone bosons, living in the coset space $G / H$, implies the vanishing of the scattering amplitude with a single soft Goldstone boson, giving rise to the famous Adler's zeros, while leading to a peculiar nonzero universal behaviour of the amplitude with two soft Goldstone particles [56, 57]. The double-soft theorems for different kinds of scalars were computed in ref. [58] using the CHY representation of the scattering amplitude [59]. The double-soft theorems for gluons were computed in refs. [60, 61] in four dimensions using the spinor helicity formalism and the BCFW recursion relations, and this result has since been confirmed in ref. [60] by a calculation in an arbitrary number of spacetime dimensions, using the CHY representation of the scattering amplitude. Ref. [60] also uses superstring theory obtaining agreement with field theory results without extra string corrections. Finally, in ref. [62] the so-called CSW method is used to compute the double-soft behavior in four dimensions.

In this work we compute the leading double-soft limit for gluons and scalars directly on the scattering amplitudes for gluons and scalars in the bosonic string. The corresponding result in the limiting field theory is then obtained by performing the field theory limit $\left(\alpha^{\prime} \rightarrow 0\right)$. Our results are valid for any number of space-time dimensions and without fixing a particular gauge. In particular, we consider scattering amplitudes of massless open strings on a $\mathrm{D} p$-brane in the bosonic string and use them to derive double-soft theorems for both gauge fields and scalars. In fact, in the presence of a $\mathrm{D} p$-brane, the original Lorentz symmetry $\mathrm{SO}(1, d-1)$ is broken into $\mathrm{SO}(1, p) \otimes \mathrm{SO}(d-1-p)$ and the original $d$-dimensional gauge field gives rise to a $(p+1)$-dimensional gauge field that we in the following, for the sake of simplicity, call gluon, and $d-p-1$ massless scalars. ${ }^{2}$ They all live on the $(p+1)$-dimensional world-volume of the $\mathrm{D} p$-brane.

We start by computing the color-ordered amplitude with $(n+2)$ gluons. We keep only the leading term when two contiguous gluons with momenta $q_{1}$ and $q_{2}$ become simultaneously soft, while the momenta $k_{1} \ldots k_{n}$ of the other gluons are kept finite. In this case, where the gluons are ordered as $k_{1} \ldots k_{n-1} q_{1} q_{2} k_{n}$, we find that the leading term in the double-soft limit behaves as $\frac{1}{q_{1,2}^{2}}$ and is given by:

$$
\begin{aligned}
M_{2 g ; n g}=\frac{g_{p+1}^{2}}{q_{1} q_{2}}\{ & -\frac{1}{2}\left(\epsilon_{q_{1}} \epsilon_{q_{2}}\right)\left[\frac{k_{n}\left(q_{2}-q_{1}\right)+q_{1} q_{2}}{k_{n}\left(q_{1}+q_{2}\right)+q_{1} q_{2}}+\frac{k_{n-1}\left(q_{1}-q_{2}\right)+q_{1} q_{2}}{k_{n-1}\left(q_{1}+q_{2}\right)+q_{1} q_{2}}\right] \\
& +\frac{\left(\epsilon_{q_{1}} q_{2}\right)\left(\epsilon_{q_{2}} k_{n}\right)-\left(\epsilon_{q_{2}} q_{1}\right)\left(\epsilon_{q_{1}} k_{n}\right)}{\left(k_{n}\left(q_{1}+q_{2}\right)+q_{1} q_{2}\right)}-\frac{\left(\epsilon_{q_{1}} q_{2}\right)\left(\epsilon_{q_{2}} k_{n-1}\right)-\left(\epsilon_{q_{2}} q_{1}\right)\left(\epsilon_{q_{1}} k_{n-1}\right)}{\left(k_{n-1}\left(q_{1}+q_{2}\right)+q_{1} q_{2}\right)} \\
& +\frac{\left(\epsilon_{q_{1}} k_{n}\right)\left(\epsilon_{q_{2}} k_{n}\right)\left(q_{1} q_{2}\right)}{\left(k_{n} q_{2}\right)\left(k_{n}\left(q_{1}+q_{2}\right)+q_{1} q_{2}\right)}+\frac{\left(\epsilon_{q_{1}} k_{n-1}\right)\left(\epsilon_{q_{2}} k_{n-1}\right)\left(q_{1} q_{2}\right)}{\left(k_{n-1} q_{1}\right)\left(k_{n-1}\left(q_{1}+q_{2}\right)+q_{1} q_{2}\right)} \\
& \left.-\frac{\left(\epsilon_{q_{1}} k_{n-1}\right)\left(\epsilon_{q_{2}} k_{n}\right)\left(q_{1} q_{2}\right)}{\left(k_{n-1} q_{1}\right)\left(k_{n} q_{2}\right)}\right\} M_{n g}
\end{aligned}
$$

\footnotetext{
${ }^{2}$ Here and in the following we keep $d$ arbitrary although for the bosonic string we need to take $d=26$.
} 
where $g_{p+1}$ is the gauge coupling constant of the gauge theory living on the $\mathrm{D} p$-brane and

$$
M_{n g}=\frac{\left(2 \alpha^{\prime}\right)^{\frac{n-2}{2}}}{\alpha^{\prime}} g_{p+1}^{n-2} \int \frac{\prod_{i=1}^{n} d z_{i}}{d V_{a b c}} \prod_{i=1}^{n} d \theta_{i}\left\langle 0\left|\prod_{i=1}^{n} \mathrm{e}^{i\left(\theta_{i} \epsilon_{i} \partial_{z_{i}}+\sqrt{2 \alpha^{\prime}} k_{i}\right) X\left(z_{i}\right)}\right| 0\right\rangle
$$

is the scattering amplitude of $n$ gluons in the bosonic string. As we explicitly show in the next sections the dependence on $\alpha^{\prime}$ in the soft factor drops out, while it is still present in the $n$-gluon amplitude. The field-theory limit, corresponding to $\alpha^{\prime} \rightarrow 0$, leaves the soft factor unchanged and acts only on $M_{n g}$ giving then just the $n$-gluon amplitude of Yang-Mills theory. Notice also that, unlike the case of a single soft behavior, the double-soft behavior cannot be written as the difference of two terms, one depending on $k_{n-1}$ and the other on $k_{n}$, because of the presence of the last term in eq. (1.1) that contains both momenta.

Eq. (1.1) reproduces eqs. (2.25) and (2.28) of ref. [60] in the gauge chosen there, namely $\left(\epsilon_{q_{2}} k_{n}\right)=\left(\epsilon_{q_{1}} k_{n-1}\right)=0$ for an arbitrary $(p+1)$-dimensional space-time. When we restrict to a four-dimensional space-time, one can use the four-dimensional helicity formalism and, in this case, one reproduces eqs. (2.1), (2.10) and (2.12) of ref. [60] and eqs. (39) and (40) of ref. [61].

We then consider the double-soft limit for two identical scalars in an amplitude with $n$ gluons and we get the following double-soft behavior:

$$
M_{2 s ; n g}=-\frac{g_{p+1}^{2}}{2 q_{1} q_{2}}\left[\frac{k_{n}\left(q_{2}-q_{1}\right)+q_{1} q_{2}}{k_{n}\left(q_{1}+q_{2}\right)+q_{1} q_{2}}+\frac{k_{n-1}\left(q_{1}-q_{2}\right)+q_{1} q_{2}}{k_{n-1}\left(q_{1}+q_{2}\right)+q_{1} q_{2}}\right] M_{n g}
$$

where $M_{n g}$ is again given in eq. (1.2). Finally, we get the same double-soft behavior for a scattering amplitude with $n+2$ identical scalars when two contiguous scalars become soft:

$$
M_{2 s ; n s}=-\frac{g_{p+1}^{2}}{2 q_{1} q_{2}}\left[\frac{k_{n}\left(q_{2}-q_{1}\right)+q_{1} q_{2}}{k_{n}\left(q_{1}+q_{2}\right)+q_{1} q_{2}}+\frac{k_{n-1}\left(q_{1}-q_{2}\right)+q_{1} q_{2}}{k_{n-1}\left(q_{1}+q_{2}\right)+q_{1} q_{2}}\right] M_{n s}
$$

where $M_{n s}$ is the $n$-scalar amplitude in the bosonic string. This equation agrees with eqs. (11) and (12) of ref. [58] for the special case of $m=-1$.

Notice that in our three main results we have kept terms involving $q_{1} q_{2}$ because they come naturally out of our calculations, ${ }^{3}$ although there are other terms of the same order that we have not computed.

The three previous formulas are valid in an arbitrary $(p+1)$-dimensional space-time and all follow from a $d$-dimensional gauge theory that, because of the presence of a $\mathrm{D} p$ brane, gives rise to a gauge theory in a $(p+1)$-dimensional space-time coupled to $(d-p-1)$ scalar fields, which correspond to the components of the original $d$-dimensional gauge field along the directions outside the world-volume of the $\mathrm{D} p$-brane. Because of this, eqs. (1.3) and (1.4) follow from eq. (1.1) by neglecting the terms where the polarizations of the soft gluons are contracted with the momenta of all particles. This is a consequence of the fact that the scalars correspond to the components of the gluons in the directions orthogonal to the $\mathrm{D} p$-brane.

\footnotetext{
${ }^{3}$ See also ref. [58].
} 
It is also interesting to note that the previous three soft amplitudes all involve the factor in the squared bracket of eqs. (1.3) and (1.4) that is typical of the double-soft limit of Goldstone bosons [56]. Unlike the case of Goldstone bosons, however, in this case we get an additional singular factor $\frac{1}{q_{1} \cdot q_{2}}$.

We have also computed the leading double-soft behavior of a color-ordered amplitude with $n+2$ gluons in the case where the two soft gluons are not next to each other as before, but have a hard gluon between them, which we take to be the one with momentum $k_{n-1}$. When the gluons are ordered as $k_{1}, k_{2} \ldots q_{1} k_{n-1} q_{2} k_{n}$, the leading double-soft behavior is given by

$$
\begin{aligned}
M_{2 g ; n g}=g_{p+1}^{2}[ & \frac{k_{n-2} \epsilon_{q_{1}}}{k_{n-2} q_{1}}\left(\frac{k_{n-1} \epsilon_{q_{2}}}{k_{n-1} q_{2}}-\frac{k_{n} \epsilon_{q_{2}}}{k_{n} q_{2}}\right)+\frac{k_{n-1} \epsilon_{q_{1}}}{k_{n-1} q_{1}} \frac{k_{n} \epsilon_{q_{2}}}{k_{n} q_{2}} \\
& \left.-\frac{\left(\epsilon_{q_{1}} k_{n-1}\right)\left(\epsilon_{q_{2}} k_{n-1}\right)}{k_{n-1}\left(q_{1}+q_{2}\right)+q_{1} q_{2}}\left(\frac{1}{k_{n-1} q_{1}}+\frac{1}{k_{n-1} q_{2}}\right)\right] M_{n g}
\end{aligned}
$$

Finally, for the color-ordered amplitude where the two soft gluons have more than one hard gluon between them, the double-soft behavior is just given by the product of two single-soft behaviors.

The paper is organized as follows. In section 2 we discuss the double-soft limit for gluons in an amplitude with $n$ gluons. Section 3 is devoted to the double-soft limit for two scalars in an amplitude with $n$ gluons, while in section 4 we discuss the leading doublesoft limit for two scalars in an amplitude with $n$ scalars. The last section is left for conclusions. In the appendix we compute the double-soft limit of some double integrals used for computing the double-soft limits.

\section{Double-soft behavior with $n+2$ gluons}

In this section we consider the color-ordered scattering amplitude involving $(n+2)$ gauge fields living on the world-volume of a $\mathrm{D} p$-brane of the bosonic string and we compute the leading double-soft behavior when two contiguous gluons become simultaneously soft.

We denote with $\left(\epsilon_{q_{1}}, q_{1}\right)$ and $\left(\epsilon_{q_{2}}, q_{2}\right)$ the polarizations and momenta of the gluons that eventually will become soft and with $\left(\epsilon_{i}, k_{i}\right)$ the polarizations and momenta of the remaining gluons. We consider the color-ordered amplitude corresponding to the following permutation: $k_{1}, k_{2}, \ldots k_{n-1}, q_{1}, q_{2}, k_{n}$ for which the Koba-Nielsen variables of the various gluons are ordered as $z_{1} \geq z_{2} \geq z_{3} \cdots \geq z_{n-1} \geq w_{1} \geq w_{2} \geq z_{n}$.

It is convenient to write the amplitude with $(n+2)$ gluons by exponentiating the derivative part of the vertex operators by introducing for each vertex operator a Grassmann variable, called $\theta_{i}, i=1 \ldots n$ and $\phi_{a}, a=1,2$, and by writing the scattering amplitude as 
follows: ${ }^{4}$

$$
\begin{aligned}
M_{2 g ; n g}= & \frac{\left(\sqrt{2 \alpha^{\prime}}\right)^{n}}{\alpha^{\prime}} g_{p+1}^{n} \int \frac{\prod_{i=1}^{n} d z_{i}}{d V_{a b c}} \prod_{i=1}^{n} d \theta_{i} \int_{0}^{z_{n-1}} d w_{1} \int_{0}^{w_{1}} d w_{2} \int d \phi_{1} d \phi_{2} \\
& \times\langle 0| \prod_{i=1}^{n-1} \mathrm{e}^{i\left(\theta_{i} \epsilon_{i} \partial_{z_{i}}+\sqrt{2 \alpha^{\prime}} k_{i}\right) X\left(z_{i}\right)} \\
& \left.\times \mathrm{e}^{i\left(\phi_{1} \epsilon_{q_{1}} \partial_{w_{1}}+\sqrt{2 \alpha^{\prime}} q_{1}\right) X\left(w_{1}\right)} \mathrm{e}^{i\left(\phi_{2} \epsilon_{q_{2}} \partial_{w_{2}}+\sqrt{2 \alpha^{\prime}} q_{2}\right) X\left(w_{2}\right)} \mathrm{e}^{i\left(\theta_{n} \epsilon_{n} \partial_{z_{n}}+\sqrt{2 \alpha^{\prime}} k_{n}\right) X\left(z_{n}\right)}\right\rangle,
\end{aligned}
$$

where $g_{p+1}$ is the $(p+1)$-dimensional gauge coupling constant. ${ }^{5}$ Using the contraction

$$
X_{\mu}(z) X_{\nu}(w) \sim-\eta_{\mu \nu} \log (z-w) \quad \text { with } \quad \eta_{\mu \nu}=(-++\cdots+)
$$

we can contract the vertex operators of the states with momenta $q_{1}$ and $q_{2}$, with those of the other states getting

$$
\begin{aligned}
& M_{2 g ; n g}=\frac{\left(\sqrt{2 \alpha^{\prime}}\right)^{n}}{\alpha^{\prime}} g_{p+1}^{n} \int \frac{\prod_{i=1}^{n} d z_{i}}{d V_{a b c}} \prod_{i=1}^{n} d \theta_{i}\left\langle 0\left|\prod_{i=1}^{n} \mathrm{e}^{i\left(\theta_{i} \epsilon_{i} \partial_{z_{i}}+\sqrt{2 \alpha^{\prime}} k_{i}\right) X\left(z_{i}\right)}\right| 0\right\rangle \\
& \times \int_{0}^{z_{n-1}} d w_{1} \int_{0}^{w_{1}} d w_{2} \int d \phi_{1} d \phi_{2} \\
& \times \prod_{i=1}^{n-1}\left(\left(z_{i}-w_{1}\right)^{2 \alpha^{\prime} k_{i} q_{1}}\left(z_{i}-w_{2}\right)^{2 \alpha^{\prime} k_{i} q_{2}}\right)\left(w_{1}-z_{n}\right)^{2 \alpha^{\prime} q_{1} k_{n}}\left(w_{2}-z_{n}\right)^{2 \alpha^{\prime} q_{2} k_{n}} \\
& \times\left(w_{1}-w_{2}\right)^{2 \alpha^{\prime} q_{1} q_{2}} \prod_{i=1}^{n}\left(\mathrm{e}^{\sqrt{2 \alpha^{\prime}} \frac{\theta_{i} \epsilon_{i} q_{1}}{z_{i}-w_{1}}} \mathrm{e}^{\sqrt{2 \alpha^{\prime}} \frac{\theta_{i} \epsilon_{i} q_{2}}{z_{i}-w_{2}}}\right) \mathrm{e}^{-\phi_{1} \phi_{2} \frac{\left(\epsilon_{q_{1}} \epsilon q_{2}\right)}{\left(w_{1}-w_{2}\right)^{2}}} \\
& \times \mathrm{e}^{\phi_{1}\left[\sum_{i=1}^{n} \frac{\theta_{i}\left(\epsilon_{q_{1}} \epsilon_{i}\right)}{\left(z_{i}-w_{1}\right)^{2}}-\sum_{i=1}^{n} \frac{\sqrt{2 \alpha^{\prime}}\left(k_{i} \epsilon_{q_{1}}\right)}{z_{i}-w_{1}}+\frac{\sqrt{2 \alpha^{\prime}}\left(\epsilon_{q_{1}} q_{2}\right)}{w_{1}-w_{2}}\right]} \\
& \times \mathrm{e}^{\phi_{2}\left[\sum_{i=1}^{n} \frac{\theta_{i}\left(\epsilon_{2} \epsilon_{i}\right)}{\left(z_{i}-w_{2}\right)^{2}}-\sum_{i=1}^{n} \frac{\sqrt{2 \alpha^{\prime}}\left(k_{i} \epsilon_{2}\right)}{z_{i}-w_{2}}-\frac{\sqrt{2 \alpha^{\prime}}\left(\epsilon_{q_{2}} q_{1}\right)}{w_{1}-w_{2}}\right] .}
\end{aligned}
$$

We can now perform the Grassmann integrals over $\phi_{1}$ and $\phi_{2}$ arriving at

$$
\begin{aligned}
M_{2 g ; n g}= & \frac{\left(2 \alpha^{\prime}\right)^{\frac{n-2}{2}}}{\alpha^{\prime}} g_{p+1}^{n-2} \int \frac{\prod_{i=1}^{n} d z_{i}}{d V_{a b c}} \prod_{i=1}^{n} d \theta_{i}\left\langle 0\left|\prod_{i=1}^{n} \mathrm{e}^{i\left(\theta_{i} \epsilon_{i} \partial_{z_{i}}+\sqrt{2 \alpha^{\prime}} k_{i}\right) X\left(z_{i}\right)}\right| 0\right\rangle \\
& \times\left(2 \alpha^{\prime} g_{p+1}^{2}\right) \int_{0}^{z_{n-1}} d w_{1} \int_{0}^{w_{1}} d w_{2} \prod_{i=1}^{n-1}\left(\left(z_{i}-w_{1}\right)^{2 \alpha^{\prime} k_{i} q_{1}}\left(z_{i}-w_{2}\right)^{2 \alpha^{\prime} k_{i} q_{2}}\right) \\
& \times\left(w_{1}-z_{n}\right)^{2 \alpha^{\prime} q_{1} k_{n}}\left(w_{2}-z_{n}\right)^{2 \alpha^{\prime} q_{2} k_{n}}\left(w_{1}-w_{2}\right)^{2 \alpha^{\prime} q_{1} q_{2}} \prod_{i=1}^{n}\left(\mathrm{e}^{\sqrt{2 \alpha^{\prime}} \frac{\theta_{i} \epsilon_{i} q_{1}}{z_{i}-w_{1}}} \mathrm{e}^{\sqrt{2 \alpha^{\prime}} \frac{\theta_{i} \epsilon_{i} q_{2}}{z_{i}-w_{2}}}\right) \\
& \times\left\{\frac{\left(\epsilon_{q_{1}} \epsilon_{q_{2}}\right)}{\left(w_{1}-w_{2}\right)^{2}}+\left[\sum_{i=1}^{n} \frac{\theta_{i}\left(\epsilon_{i} \epsilon_{q_{1}}\right)}{\left(z_{i}-w_{1}\right)^{2}}-\sum_{i=1}^{n} \frac{\sqrt{2 \alpha^{\prime}}\left(k_{i} \epsilon_{q_{1}}\right)}{z_{i}-w_{1}}+\frac{\sqrt{2 \alpha^{\prime}}\left(\epsilon_{q_{1}} q_{2}\right)}{w_{1}-w_{2}}\right]\right. \\
& \left.\times\left[\sum_{j=1}^{n} \frac{\theta_{j}\left(\epsilon_{j} \epsilon_{q_{2}}\right)}{\left(z_{j}-w_{2}\right)^{2}}-\sum_{j=1}^{n} \frac{\sqrt{2 \alpha^{\prime}}\left(k_{j} \epsilon_{q_{2}}\right)}{z_{j}-w_{2}}-\frac{\sqrt{2 \alpha^{\prime}}\left(\epsilon_{q_{2}} q_{1}\right)}{w_{1}-w_{2}}\right]\right\} \equiv M_{n g} * G_{n},
\end{aligned}
$$

\footnotetext{
${ }^{4}$ Here and in the following we assume that $z_{n}=0$.

${ }^{5}$ In order to have a bosonic quantity in the exponents, we assume that also the polarization vectors are Grassmann variables.
} 
where by $*$ a convolution of the integrals is understood,

$$
M_{n g}=\frac{\left(2 \alpha^{\prime}\right)^{\frac{n-2}{2}}}{\alpha^{\prime}} g_{p+1}^{n-2} \int \frac{\prod_{i=1}^{n} d z_{i}}{d V_{a b c}} \prod_{i=1}^{n} d \theta_{i}\left\langle 0\left|\prod_{i=1}^{n} \mathrm{e}^{i\left(\theta_{i} \epsilon_{i} \partial_{z_{i}}+\sqrt{2 \alpha^{\prime}} k_{i}\right) X\left(z_{i}\right)}\right| 0\right\rangle
$$

is the scattering amplitude of $n$ gluons, and

$$
\begin{aligned}
G_{n}= & \left(2 \alpha^{\prime} g_{p+1}^{2}\right) \int_{0}^{z_{n-1}} d w_{1} \int_{0}^{w_{1}} d w_{2} \prod_{i=1}^{n}\left(\left(z_{i}-w_{1}\right)^{2 \alpha^{\prime} k_{i} q_{1}}\left(z_{i}-w_{2}\right)^{2 \alpha^{\prime} k_{i} q_{2}}\right) \\
& \times\left(w_{1}-w_{2}\right)^{2 \alpha^{\prime} q_{1} q_{2}} \prod_{i=1}^{n}\left(\mathrm{e}^{\sqrt{2 \alpha^{\prime}} \frac{\theta_{i} \epsilon_{i} q_{1}}{z_{i}-w_{1}}} \mathrm{e}^{\sqrt{2 \alpha^{\prime}} \frac{\theta_{i} \epsilon_{i} q_{2}}{z_{i}-w_{2}}}\right) \\
& \times\left\{\frac{\left(\epsilon_{q_{1}} \epsilon_{q_{2}}\right)}{\left(w_{1}-w_{2}\right)^{2}}+\left[\sum_{i=1}^{n} \frac{\theta_{i}\left(\epsilon_{i} \epsilon_{q_{1}}\right)}{\left(z_{i}-w_{1}\right)^{2}}-\sum_{i=1}^{n} \frac{\sqrt{2 \alpha^{\prime}}\left(k_{i} \epsilon_{q_{1}}\right)}{z_{i}-w_{1}}+\frac{\sqrt{2 \alpha^{\prime}}\left(\epsilon_{q_{1}} q_{2}\right)}{w_{1}-w_{2}}\right]\right. \\
& \left.\times\left[\sum_{j=1}^{n} \frac{\theta_{j}\left(\epsilon_{j} \epsilon_{q_{2}}\right)}{\left(z_{j}-w_{2}\right)^{2}}-\sum_{j=1}^{n} \frac{\sqrt{2 \alpha^{\prime}}\left(k_{j} \epsilon_{q_{2}}\right)}{z_{j}-w_{2}}-\frac{\sqrt{2 \alpha^{\prime}}\left(\epsilon_{q_{2}} q_{1}\right)}{w_{1}-w_{2}}\right]\right\} .
\end{aligned}
$$

This expression contains two kinds of terms. The first one is without any dependence on the variables $\theta_{i}$ and acts in the convolution at the end of eq. (2.4) as a factor that multiplies the $n$-gluon amplitude $M_{n g}$, while the second one contains the terms with $\theta_{i}$ that, when acting in the convolution in eq. (2.4), modify the structure of the $n$-gluon amplitude $M_{n g}$. It can be shown that the first kind of terms is the leading one in the double-soft limit, while the second one is subleading. In this paper we compute only the leading one that is equal to

$$
\begin{aligned}
& G_{n}^{(1)}=g_{p+1}^{2}\left(2 \alpha^{\prime}\right) \int_{0}^{z_{n-1}} d w_{1} \int_{0}^{w_{1}} d w_{2}\left(w_{1}-w_{2}\right)^{2 \alpha^{\prime} q_{1} \cdot q_{2}} w_{1}^{2 \alpha^{\prime} q_{1} \cdot k_{n}} w_{2}^{2 \alpha^{\prime} q_{2} \cdot k_{n}} \\
& \times\left(z_{n-1}-w_{1}\right)^{2 \alpha^{\prime} q_{1} \cdot k_{n-1}}\left(z_{n-1}-w_{2}\right)^{2 \alpha^{\prime} q_{2} \cdot k_{n-1}} \\
& \times\left\{\frac{\epsilon_{q_{1}} \cdot \epsilon_{q_{2}}}{\left(w_{1}-w_{2}\right)^{2}}+\left[\frac{\sqrt{2 \alpha^{\prime}}\left(k_{n-1} \epsilon_{q_{1}}\right)}{w_{1}-z_{n-1}}+\frac{\sqrt{2 \alpha^{\prime}}\left(k_{n} \epsilon_{q_{1}}\right)}{w_{1}}+\frac{\sqrt{2 \alpha^{\prime}}\left(q_{2} \epsilon_{q_{1}}\right)}{w_{1}-w_{2}}\right]\right. \\
& \left.\times\left[\frac{\sqrt{2 \alpha^{\prime}}\left(k_{n-1} \epsilon_{q_{2}}\right)}{w_{2}-z_{n-1}}+\frac{\sqrt{2 \alpha^{\prime}}\left(k_{n} \epsilon_{q_{2}}\right)}{w_{2}}+\frac{\sqrt{2 \alpha^{\prime}}\left(q_{1} \epsilon_{q_{2}}\right)}{w_{2}-w_{1}}\right]\right\}
\end{aligned}
$$

It is convenient to introduce two new variables $z_{n-1} \hat{w}_{a}=w_{a}$ for $a=1,2$. We get

$$
\begin{aligned}
G_{n}^{(1)}= & g_{p+1}^{2}\left(2 \alpha^{\prime}\right) z_{n-1}^{2 \alpha^{\prime}\left[q_{1} q_{2}+\left(k_{n-1}+k_{n}\right)\left(q_{1}+q_{2}\right)\right]} \int_{0}^{1} d \hat{w}_{1} \int_{0}^{\hat{w}_{1}} d \hat{w}_{2}\left(\hat{w}_{1}-\hat{w}_{2}\right)^{2 \alpha^{\prime} q_{1} \cdot q_{2}} \\
& \times \hat{w}_{1}^{2 \alpha^{\prime} q_{1} \cdot k_{n}} \hat{w}_{2}^{2 \alpha^{\prime} q_{2} \cdot k_{n}}\left(1-\hat{w}_{1}\right)^{2 \alpha^{\prime} q_{1} \cdot k_{n-1}}\left(1-\hat{w}_{2}\right)^{2 \alpha^{\prime} q_{2} \cdot k_{n-1}} \\
& \times\left\{\frac{\epsilon_{q_{1}} \cdot \epsilon_{q_{2}}}{\left(\hat{w}_{1}-\hat{w}_{2}\right)^{2}}+\left[-\frac{\sqrt{2 \alpha^{\prime}}\left(k_{n-1} \epsilon_{q_{1}}\right)}{1-\hat{w}_{1}}+\frac{\sqrt{2 \alpha^{\prime}}\left(k_{n} \epsilon_{q_{1}}\right)}{\hat{w}_{1}}+\frac{\sqrt{2 \alpha^{\prime}}\left(q_{2} \epsilon_{q_{1}}\right)}{\hat{w}_{1}-\hat{w}_{2}}\right]\right. \\
& \left.\times\left[-\frac{\sqrt{2 \alpha^{\prime}}\left(k_{n-1} \epsilon_{q_{2}}\right)}{1-\hat{w}_{2}}+\frac{\sqrt{2 \alpha^{\prime}}\left(k_{n} \epsilon_{q_{2}}\right)}{\hat{w}_{2}}-\frac{\sqrt{2 \alpha^{\prime}}\left(q_{1} \epsilon_{q_{2}}\right)}{\hat{w}_{1}-\hat{w}_{2}}\right]\right\}
\end{aligned}
$$

We want to extract from the previous sum of integrals the most singular term in the double-soft limit. In this limit, we can approximate $z_{n-1}^{2 \alpha^{\prime}\left[q_{1} q_{2}+\left(k_{n-1}+k_{n}\right)\left(q_{1}+q_{2}\right)\right]}$ with 1 . The 
double-soft behavior of the various integrals is computed in appendix A. Here we give only the final result:

$$
\begin{aligned}
G_{n}^{(1)}=g_{p+1}^{2}\{ & -\frac{\left(\epsilon_{q_{1}} \epsilon_{q_{2}}\right)}{2 q_{1} q_{2}}\left[\frac{k_{n}\left(q_{2}-q_{1}\right)+q_{1} q_{2}}{k_{n}\left(q_{1}+q_{2}\right)+q_{1} q_{2}}+\frac{k_{n-1}\left(q_{1}-q_{2}\right)+q_{1} q_{2}}{k_{n-1}\left(q_{1}+q_{2}\right)+q_{1} q_{2}}\right] \\
& \times \frac{1}{q_{1} q_{2}}\left[\frac{\left(\epsilon_{q_{1}} q_{2}\right)\left(\epsilon_{q_{2}} k_{n}\right)-\left(\epsilon_{q_{2}} q_{1}\right)\left(\epsilon_{q_{1}} k_{n}\right)}{k_{n}\left(q_{1}+q_{2}\right)+q_{1} q_{2}}-\frac{\left(\epsilon_{q_{1}} q_{2}\right)\left(\epsilon_{q_{2}} k_{n-1}\right)-\left(\epsilon_{q_{2}} q_{1}\right)\left(\epsilon_{q_{1}} k_{n-1}\right)}{k_{n-1}\left(q_{1}+q_{2}\right)+q_{1} q_{2}}\right] \\
& +\frac{\left(\epsilon_{q_{1}} k_{n}\right)\left(\epsilon_{q_{2}} k_{n}\right)}{q_{2} k_{n}\left[k_{n}\left(q_{1}+q_{2}\right)+q_{1} q_{2}\right]}+\frac{\left(\epsilon_{q_{1}} k_{n-1}\right)\left(\epsilon_{q_{2}} k_{n-1}\right)}{q_{1} k_{n-1}\left[k_{n-1}\left(q_{1}+q_{2}\right)+q_{1} \cdot q_{2}\right]} \\
& \left.-\frac{\left(\epsilon_{q_{1}} k_{n-1}\right)\left(\epsilon_{q_{2}} k_{n}\right)}{\left(q_{1} k_{n-1}\right)\left(q_{2} k_{n}\right)}\right\} .
\end{aligned}
$$

In conclusion, the leading double-soft behavior is given by:

$$
\begin{aligned}
M_{2 g ; n g}=\frac{g_{p+1}^{2}}{q_{1} q_{2}}\{ & -\frac{1}{2}\left(\epsilon_{q_{1}} \epsilon_{q_{2}}\right)\left[\frac{k_{n}\left(q_{2}-q_{1}\right)+q_{1} q_{2}}{k_{n}\left(q_{1}+q_{2}\right)+q_{1} q_{2}}+\frac{k_{n-1}\left(q_{1}-q_{2}\right)+q_{1} q_{2}}{k_{n-1}\left(q_{1}+q_{2}\right)+q_{1} q_{2}}\right] \\
& +\frac{\left(\epsilon_{q_{1}} q_{2}\right)\left(\epsilon_{q_{2}} k_{n}\right)-\left(\epsilon_{q_{2}} q_{1}\right)\left(\epsilon_{q_{1}} k_{n}\right)}{\left(k_{n}\left(q_{1}+q_{2}\right)+q_{1} q_{2}\right)}-\frac{\left(\epsilon_{q_{1}} q_{2}\right)\left(\epsilon_{q_{2}} k_{n-1}\right)-\left(\epsilon_{q_{2}} q_{1}\right)\left(\epsilon_{q_{1}} k_{n-1}\right)}{\left(k_{n-1}\left(q_{1}+q_{2}\right)+q_{1} q_{2}\right)} \\
& +\frac{\left(\epsilon_{q_{1}} k_{n}\right)\left(\epsilon_{q_{2}} k_{n}\right)\left(q_{1} q_{2}\right)}{\left(k_{n} q_{2}\right)\left(k_{n}\left(q_{1}+q_{2}\right)+q_{1} q_{2}\right)}+\frac{\left(\epsilon_{q_{1}} k_{n-1}\right)\left(\epsilon_{q_{2}} k_{n-1}\right)\left(q_{1} q_{2}\right)}{\left(k_{n-1} q_{1}\right)\left(k_{n-1}\left(q_{1}+q_{2}\right)+q_{1} q_{2}\right)} \\
& \left.-\frac{\left(\epsilon_{q_{1}} k_{n-1}\right)\left(\epsilon_{q_{2}} k_{n}\right)\left(q_{1} q_{2}\right)}{\left(k_{n-1} q_{1}\right)\left(k_{n} q_{2}\right)}\right\} M_{n g},
\end{aligned}
$$

which behaves as $\frac{1}{q_{1} q_{2}}$ in the double-soft limit, i.e. when both $q_{1}$ and $q_{2}$ simultaneously go to zero. $M_{n g}$ is the $n$-gluon amplitude in the bosonic string. We see that, in the double-soft limit, the dependence on $\alpha^{\prime}$ of the soft factor in eq. (2.9) cancels, while it is still kept in the $n$-gluon amplitude in eq. (2.5).

One can check gauge invariance of the soft factor in eq. (2.9) by substituting $\epsilon_{q_{1}}$ with $q_{1}$. One gets:

$$
-g_{p+1}^{2}\left(\frac{\left(q_{1} \epsilon_{q_{2}}\right)}{k_{n-1}\left(q_{1}+q_{2}\right)+q_{1} q_{2}}+\frac{\left(q_{1} q_{2}\right)\left(\epsilon_{q_{2}} k_{n}\right)}{\left(k_{n} q_{2}\right)\left(k_{n}\left(q_{1}+q_{2}\right)+q_{1} q_{2}\right)}\right) \sim q_{1,2}^{0} .
$$

The original amplitude behaves as $\frac{1}{q_{1.2}^{2}}$. Saturating with $q_{1}$, we find that the terms of order $\frac{1}{q_{1,2}}$ cancel and we are left with a term of order $q_{1,2}^{0}$ that should be cancelled by the next to the leading term, which we have not yet computed.

One can also check gauge invariance with respect to the other soft particle by substituting $\epsilon_{q_{2}}$ with $q_{2}$. One gets:

$$
-g_{p+1}^{2}\left(\frac{\left(\epsilon_{q_{1}} q_{2}\right)}{k_{n}\left(q_{1}+q_{2}\right)+q_{1} q_{2}}+\frac{\left(\epsilon_{q_{1}} k_{n-1}\right)\left(q_{1} q_{2}\right)}{\left(k_{n-1}\left(q_{1}+q_{2}\right)+q_{1} q_{2}\right)\left(k_{n-1} q_{1}\right)}\right)
$$

which is equal to the expression in eq. (2.11) by the substitutions $k_{n-1} \leftrightarrow k_{n}$ and $q_{1} \leftrightarrow q_{2}$. 
In order to see the pole structure of the amplitude, it is convenient to write eq. (2.10) as follows:

$$
\begin{aligned}
M_{2 g ; n g}=\frac{2 g_{p+1}^{2}}{\left(q_{1}+q_{2}\right)^{2}}\{[ & -\left(\epsilon_{q_{1}} \epsilon_{q_{2}}\right) \frac{k_{n}\left(q_{2}-q_{1}\right)+q_{1} q_{2}}{\left(k_{n}+q_{1}+q_{2}\right)^{2}}+2 \frac{\left(\epsilon_{q_{1}} q_{2}\right)\left(\epsilon_{q_{2}} k_{n}\right)-\left(\epsilon_{q_{2}} q_{1}\right)\left(\epsilon_{q_{1}} k_{n}\right)}{\left(k_{n}+q_{1}+q_{2}\right)^{2}} \\
& \left.+2 \frac{\left(\epsilon_{q_{1}} k_{n}\right)\left(\epsilon_{q_{2}} k_{n}\right)\left(q_{1}+q_{2}\right)^{2}}{\left(k_{n}+q_{2}\right)^{2}\left(k_{n}+q_{1}+q_{2}\right)^{2}}\right] \\
& -\left[-\left(\epsilon_{q_{2}} \epsilon_{q_{1}}\right) \frac{k_{n-1}\left(q_{1}-q_{2}\right)+q_{1} q_{2}}{\left(k_{n-1}+q_{1}+q_{2}\right)^{2}}+2 \frac{\left(\epsilon_{q_{1}} q_{2}\right)\left(\epsilon_{q_{2}} k_{n-1}\right)-\left(\epsilon_{q_{2}} q_{1}\right)\left(\epsilon_{q_{1}} k_{n-1}\right)}{\left(k_{n-1}+q_{1}+q_{2}\right)^{2}}\right. \\
& \left.\left.+2 \frac{\left(\epsilon_{q_{1}} k_{n-1}\right)\left(\epsilon_{q_{2}} k_{n-1}\right)\left(q_{1}+q_{2}\right)^{2}}{\left(k_{n-1}+q_{1}\right)^{2}\left(k_{n-1}+q_{1}+q_{2}\right)^{2}}\right]-2 \frac{\left(\epsilon_{q_{1}} k_{n-1}\right)\left(\epsilon_{q_{2}} k_{n}\right)\left(q_{1}+q_{2}\right)^{2}}{\left(k_{n-1}+q_{1}\right)^{2}\left(k_{n}+q_{2}\right)^{2}}\right\} M_{n g}
\end{aligned}
$$

This expression shows a non-trivial pole structure and the various pole singularities are all consistent with the structure of a color-ordered amplitude. In figure 1 we sketch the various pole structures appearing in the previous equation. Notice that the final expression in eq. (2.13) is symmetric under the simultaneous change of $k_{n-1} \leftrightarrow k_{n}$ and $q_{1} \leftrightarrow q_{2}$.

Finally, eq. (2.10) reproduces eqs. (2.25) and (2.28) of ref. [60] in the gauge chosen there, namely $\left(\epsilon_{q_{2}} k_{n}\right)=\left(\epsilon_{q_{1}} k_{n-1}\right)=0$.

In the final part of this section we discuss the case of a color-ordered amplitude where the two soft gluons are not next to each other. In particular, we consider the color-ordered amplitude where a hard gluon, say the one with momentum $k_{n-1}$, is between the two soft. The gluons are then ordered as $k_{1} k_{2} \ldots k_{n-2} q_{1} k_{n-1} q_{2} k_{n}$. The complete amplitude is equal to

$$
\begin{aligned}
M_{2 g ; n g}= & \frac{\left(2 \alpha^{\prime}\right)^{\frac{n-2}{2}}}{\alpha^{\prime}} g_{p+1}^{n-2} \int \frac{\prod_{i=1}^{n-2} d z_{i} d z_{n}}{d V_{a b c}} \prod_{i=1}^{n} d \theta_{i}\left\langle 0\left|\prod_{i=1}^{n} \mathrm{e}^{i\left(\theta_{i} \epsilon \partial_{z_{i}}+\sqrt{2 \alpha^{\prime} k_{i}}\right) X\left(z_{i}\right)}\right| 0\right\rangle\left(2 \alpha^{\prime} g_{p+1}^{2}\right) \\
& \times \int_{0}^{z_{n-2}} d w_{1} \int_{0}^{w_{1}} d z_{n-1} \int_{0}^{z_{n-1}} d w_{2} \prod_{i=1}^{n-3}\left(z_{i}-w_{1}\right)^{2 \alpha^{\prime} k_{i} q_{1}} \prod_{i=1}^{n-2}\left(z_{i}-w_{2}\right)^{2 \alpha^{\prime} k_{i} q_{2}}\left(z_{n-2}-w_{1}\right)^{2 \alpha^{\prime} q_{1} \cdot k_{n-2}} \\
& \times\left(w_{1}-z_{n-1}\right)^{2 \alpha^{\prime} q_{1} k_{n-1}}\left(z_{n-1}-w_{2}\right)^{2 \alpha^{\prime} q_{2} k_{n-1}} w_{1}^{2 \alpha^{\prime} q_{1} \cdot k_{n}} w_{2}^{2 \alpha^{\prime} q_{2} \cdot k_{n}}\left(w_{1}-w_{2}\right)^{2 \alpha^{\prime} q_{1} q_{2}} \\
& \times \prod_{i=1}^{n}\left(\mathrm{e}^{\sqrt{2 \alpha^{\prime}} \frac{\theta_{i} \epsilon_{i} q_{1}}{z_{i}-w_{1}}} \mathrm{e}^{\sqrt{2 \alpha^{\prime}} \frac{\theta_{i} \epsilon_{i} q_{2}}{z_{i}-w_{2}}}\right) \\
& \times\left\{\frac{\left(\epsilon_{q_{1}} \epsilon_{q_{2}}\right)}{\left(w_{1}-w_{2}\right)^{2}}+\left[\sum_{i=1}^{n} \frac{\theta_{i}\left(\epsilon_{i} \epsilon_{q_{1}}\right)}{\left(z_{i}-w_{1}\right)^{2}}-\sum_{i=1}^{n-2} \frac{\sqrt{2 \alpha^{\prime}}\left(k_{i} \epsilon_{q_{1}}\right)}{z_{i}-w_{1}}\right.\right. \\
& \left.\quad+\frac{\sqrt{2 \alpha^{\prime}}\left(k_{n-1} \epsilon_{q_{1}}\right)}{w_{1}-z_{n-1}}+\frac{\sqrt{2 \alpha^{\prime}}\left(k_{n} \epsilon_{q_{1}}\right)}{w_{1}}+\frac{\sqrt{2 \alpha^{\prime}}\left(\epsilon_{q_{1}} q_{2}\right)}{w_{1}-w_{2}}\right] \\
& \left.\times\left[\sum_{j=1}^{n} \frac{\theta_{j}\left(\epsilon_{j} \epsilon_{q_{2}}\right)}{\left(z_{j}-w_{2}\right)^{2}}-\sum_{j=1}^{n-1} \frac{\sqrt{2 \alpha^{\prime}}\left(k_{j} \epsilon_{q_{2}}\right)}{z_{j}-w_{2}}+\frac{\sqrt{2 \alpha^{\prime}}\left(k_{n} \epsilon_{q_{2}}\right)}{w_{2}}-\frac{\sqrt{2 \alpha^{\prime}}\left(\epsilon_{q_{2}} q_{1}\right)}{w_{1}-w_{2}}\right]\right\} .
\end{aligned}
$$

Since we are interested in the leading double-soft behavior, we can neglect the terms with $\theta_{i}$ in the last four lines of the previous expression and keep in the curly bracket only the terms with momenta. It can be seen that the leading double-soft behavior is obtained by 

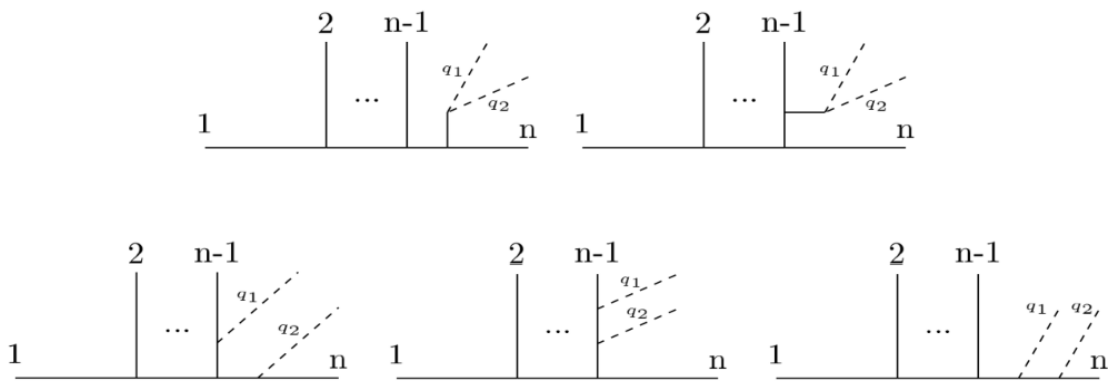

Figure 1. Sketchy diagrams of the color-ordered $n+2$ gluon amplitude, where solid lines are the hard gluons and dashed lines are the soft gluons. Each term in eq. (2.13) corresponds to one of the diagrams, and shows that all poles allowed in the color-ordered amplitude contribute to the double-soft limit.

restricting ourselves to the following expression:

$$
\begin{aligned}
& M_{2 g ; n g}=\frac{\left(2 \alpha^{\prime}\right)^{\frac{n-2}{2}}}{\alpha^{\prime}} g_{p+1}^{n-2} \int \frac{\prod_{i=1}^{n-2} d z_{i} d z_{n}}{d V_{a b c}} \prod_{i=1}^{n} d \theta_{i}\left\langle 0\left|\prod_{i=1}^{n} \mathrm{e}^{i\left(\theta_{i} \epsilon \partial_{z_{i}}+\sqrt{2 \alpha^{\prime}} k_{i}\right) X\left(z_{i}\right)}\right| 0\right\rangle \\
& \times\left(2 \alpha^{\prime}\right)^{2} g_{p+1}^{2} \int_{0}^{z_{n-2}} d w_{1} \int_{0}^{w_{1}} d z_{n-1} \int_{0}^{z_{n-1}} d w_{2}\left(z_{n-2}-w_{1}\right)^{2 \alpha^{\prime} q_{1} \cdot k_{n-2}} \\
& \times\left(w_{1}-z_{n-1}\right)^{2 \alpha^{\prime} q_{1} k_{n-1}}\left(z_{n-1}-w_{2}\right)^{2 \alpha^{\prime} q_{2} k_{n-1}} w_{2}^{2 \alpha^{\prime} q_{2} \cdot k_{n}}\left(w_{1}-w_{2}\right)^{2 \alpha^{\prime} q_{1} q_{2}} \\
& \times\left[-\frac{\left(k_{n-2} \epsilon_{q_{1}}\right)}{z_{n-2}-w_{1}}+\frac{\left(k_{n-1} \epsilon_{q_{1}}\right)}{w_{1}-z_{n-1}}\right]\left[-\frac{\left(k_{n-1} \epsilon_{q_{2}}\right)}{z_{n-1}-w_{2}}+\frac{\left(k_{n} \epsilon_{q_{2}}\right)}{w_{2}}\right] .
\end{aligned}
$$

In order to study the double-soft behavior of the four terms that appear in the last line of the previous equation, it is convenient to go to the variables $x_{i}$ with $i=1,2,3$ that run from 0 to 1 :

$$
w_{1}=z_{n-2} x_{1} ; \quad z_{n-1}=z_{n-2} x_{1} x_{2} ; \quad w_{2}=z_{n-2} x_{1} x_{2}
$$

The Jacobian of the transformation is equal to $z_{n-2}^{3} x_{1}^{2} x_{2}$ and the last three lines of eq. (2.15) become:

$$
\begin{aligned}
\left(2 \alpha^{\prime}\right)^{2} & g_{p+1}^{2} \int_{0}^{1} d x_{1} \int_{0}^{1} d x_{2} \int_{0}^{1} d x_{3}\left[z_{n-2}\left(1-x_{1}\right)\right]^{2 \alpha^{\prime} q_{1} k_{n-2}}\left[z_{n-2} x_{1}\left(1-x_{2}\right)\right]^{2 \alpha^{\prime} q_{1} k_{n-1}} \\
& \times\left[z_{n-2} x_{1} x_{2}\left(1-x_{3}\right)\right]^{2 \alpha^{\prime} q_{2} k_{n-1}}\left[z_{n-2} x_{1} x_{2} x_{3}\right]^{2 \alpha^{\prime} q_{2} k_{n}}\left[z_{n-2} x_{1}\left(1-x_{2} x_{3}\right)\right]^{2 \alpha^{\prime} q_{1} q_{2}} \\
& \times z_{n-2}\left[-x_{1} \frac{k_{n-2} \epsilon_{q_{1}}}{1-x_{1}}+\frac{k_{n-1} \epsilon_{q_{1}}}{1-x_{2}}\right]\left[-\frac{k_{n-1} \epsilon_{q_{2}}}{1-x_{3}}+\frac{k_{n} \epsilon_{q_{2}}}{x_{3}}\right]
\end{aligned}
$$

Let us consider the first term of the square bracket in the last line of the previous equation. The leading double-soft limit is obtained for $x_{1} \sim 1$ and $x_{3} \sim 1$ and $x_{3} \sim 0$ for the two terms in the second square bracket, respectively. In this corner of the integration region $z_{n-1} \equiv x_{2} z_{n-2}$ and therefore we get

$$
\begin{gathered}
\left(2 \alpha^{\prime}\right)^{2} g_{p+1}^{2} z_{n-2} \int_{0}^{1} d x_{2} \frac{k_{n-2} \epsilon_{q_{1}}}{2 \alpha^{\prime} q_{1} k_{n-2}}\left[\frac{k_{n-1} \epsilon_{q_{2}}}{2 \alpha^{\prime} q_{2} k_{n-1}}-\frac{k_{n} \epsilon_{q_{2}}}{2 \alpha^{\prime} q_{2} k_{n}}\right] \\
=g_{p+1}^{2} \frac{k_{n-2} \epsilon_{q_{1}}}{q_{1} k_{n-2}}\left[\frac{k_{n-1} \epsilon_{q_{2}}}{q_{2} k_{n-1}}-\frac{k_{n} \epsilon_{q_{2}}}{q_{2} k_{n}}\right] \int_{0}^{z_{n-2}} d z_{n-1}
\end{gathered}
$$


When multiplied with the first line of eq. (2.15), one gets the two soft terms in eq. (2.18) times the amplitude with $n$ gluons.

The leading double-soft behavior of the product of the second terms of the two square brackets in the last line of eq. (2.17) is obtained for $x_{2} \sim 1$ and $x_{3} \sim 0$. Therefore, one gets:

$$
\left(2 \alpha^{\prime}\right)^{2} g_{p+1}^{2} z_{n-2} \int_{0}^{1} d x_{1} \frac{k_{n-1} \epsilon_{q_{1}}}{2 \alpha^{\prime} k_{n-1} q_{1}} \frac{k_{n} \epsilon_{q_{2}}}{2 \alpha^{\prime} k_{n} q_{2}}=g_{p+1}^{2} \frac{k_{n-1} \epsilon_{q_{1}}}{k_{n-1} q_{1}} \frac{k_{n} \epsilon_{q_{2}}}{k_{n} q_{2}} \int_{0}^{z_{n-2}} d z_{n-1}
$$

where we have used that, for $x_{2} \sim 1$, we can write $z_{n-1}=z_{n-2} x_{1}$. When multiplied with the first line of eq. (2.15), one gets again the soft term in eq. (2.19) times the amplitude with $n$ gluons.

The leading double-soft behavior of the last term is a bit more complicated to extract. The relevant terms of eq. (2.17) that one needs to consider, are:

$$
\begin{gathered}
-\left(2 \alpha^{\prime}\right)^{2} g_{p+1}^{2} z_{n-2}\left(k_{n-1} \epsilon_{q_{1}}\right)\left(k_{n-1} \epsilon_{q_{2}}\right) \int_{0}^{1} d x_{1} \int_{0}^{1} d x_{2} x_{2}^{2 \alpha^{\prime}\left(k_{n-1}+k_{n}\right) q_{2}}\left(1-x_{2}\right)^{2 \alpha^{\prime} q_{1} k_{n-1}-1} \\
\quad \times \int_{0}^{1} d x_{3}\left(1-x_{3}\right)^{2 \alpha^{\prime} q_{2} k_{n-1}-1} x_{3}^{2 \alpha^{\prime} q_{2} k_{n}}\left(1-x_{2} x_{3}\right)^{2 \alpha^{\prime} q_{1} q_{2}}
\end{gathered}
$$

The dominant contribution comes from the region of the integrals around $x_{2} \sim 1$ and $x_{3} \sim 1$. It is convenient to first change variables to $y_{i}=1-x_{i}$ for $i=2,3$ and then to $y_{2}=t u$ and $y_{3}=t(1-u)$ with Jacobian equal to $t$. Then, eq. (2.20) becomes

$$
\begin{aligned}
-\left(2 \alpha^{\prime}\right)^{2} g_{p+1}^{2} z_{n-2}\left(k_{n-1} \epsilon_{q_{1}}\right)\left(k_{n-1} \epsilon_{q_{2}}\right) \int_{0}^{1} d x_{1} \int_{0}^{\epsilon} d t t^{2 \alpha^{\prime}\left(k_{n-1}\left(q_{1}+q_{2}\right)+q_{1} q_{2}\right)-1} \\
\times \int_{0}^{1} d u u^{2 \alpha^{\prime} k_{n-1} q_{1}-1}(1-u)^{2 \alpha^{\prime} q_{2} k_{n-1}-1}(1-t u)^{2 \alpha^{\prime} q_{2}\left(k_{n}+k_{n-1}\right)}(1-t(1-u))^{2 \alpha^{\prime} k_{n} q_{2}} \\
=-\left(2 \alpha^{\prime}\right)^{2} g_{p+1}^{2} z_{n-2}\left(k_{n-1} \epsilon_{q_{1}}\right)\left(k_{n-1} \epsilon_{q_{2}}\right) \int_{0}^{1} d x_{1} \frac{1}{2 \alpha^{\prime}\left(k_{n-1}\left(q_{1}+q_{2}\right)+q_{1} q_{2}\right)} \\
\times\left[\frac{1}{2 \alpha^{\prime} k_{n-1} q_{1}}+\frac{1}{2 \alpha^{\prime} k_{n-1} q_{2}}\right]
\end{aligned}
$$

where we have neglected irrelevant factors that go to 1 in the double-soft limit and, by introducing a cutoff $\epsilon$, we have restricted the region of integration for $t$ to a small interval around $t=0$. Eq. (2.21) is equal to

$$
-g_{p+1}^{2} \int_{0}^{z_{n-2}} d z_{n-1} \frac{\left(k_{n-1} \epsilon_{q_{1}}\right)\left(k_{n-1} \epsilon_{q_{2}}\right)}{\left(k_{n-1}\left(q_{1}+q_{2}\right)+q_{1} q_{2}\right)}\left[\frac{1}{k_{n-1} q_{1}}+\frac{1}{k_{n-1} q_{2}}\right]
$$

where, for $x_{2} \sim 1$, we have used that $z_{n-1}=z_{n-2} x_{1}$. When we insert the result in eq. (2.22) in eq. (2.17), we get again the double-soft factor times the amplitude with $n$ gluons.

In conclusion, the double-soft behavior is given by the sum of the previous three terms:

$$
\begin{aligned}
M_{2 g ; n g}=g_{p+1}^{2}[ & \frac{k_{n-2} \epsilon_{q_{1}}}{k_{n-2} q_{1}}\left(\frac{k_{n-1} \epsilon_{q_{2}}}{k_{n-1} q_{2}}-\frac{k_{n} \epsilon_{q_{2}}}{k_{n} q_{2}}\right)+\frac{k_{n-1} \epsilon_{q_{1}}}{k_{n-1} q_{1}} \frac{k_{n} \epsilon_{q_{2}}}{k_{n} q_{2}} \\
& \left.-\frac{\left(\epsilon_{q_{1}} k_{n-1}\right)\left(\epsilon_{q_{2}} k_{n-1}\right)}{k_{n-1}\left(q_{1}+q_{2}\right)+q_{1} q_{2}}\left(\frac{1}{k_{n-1} q_{1}}+\frac{1}{k_{n-1} q_{2}}\right)\right] M_{n g}
\end{aligned}
$$


It is easy to check that the soft factor is gauge invariant up to terms of order $q_{1,2}^{0}$ as in the case of two contiguous soft gluons.

Finally, the double-soft behavior when the two soft gluons are separated by more than one leg is just the product of two single soft behaviors.

\section{Double-soft behavior with 2 scalars and $n$ gluons}

In this section we compute the double-soft behavior of two scalars in an amplitude with $n$ gluons. The amplitude with two scalars and $n$ gluons can be obtained from that computed in the previous section by noticing that, in this case, we have to impose:

$$
\left(\epsilon_{i} \epsilon_{q_{1}}\right)=\left(\epsilon_{i} \epsilon_{q_{2}}\right)=\left(\epsilon_{q_{1}} k_{i}\right)=\left(\epsilon_{q_{2}} k_{i}\right)=\left(\epsilon_{q_{1}} q_{2}\right)=\left(\epsilon_{q_{2}} q_{1}\right)=0 .
$$

We then get

$$
M_{2 s ; n g}=M_{n g} * H_{n}
$$

where $M_{n g}$ is given in eq. (2.5) and

$$
\begin{aligned}
H_{n}= & \left(2 \alpha^{\prime} g_{p+1}^{2}\right) \int_{0}^{z_{n-1}} d w_{1} \int_{0}^{w_{1}} d w_{2} \prod_{i=1}^{n}\left(\left|z_{i}-w_{1}\right|^{2 \alpha^{\prime} k_{i} q_{1}}\left|z_{i}-w_{2}\right|^{2 \alpha^{\prime} k_{i} q_{2}}\right) \\
& \times\left(w_{1}-w_{2}\right)^{2 \alpha^{\prime} q_{1} q_{2}} \prod_{i=1}^{n}\left(\mathrm{e}^{\sqrt{2 \alpha^{\prime}} \frac{\theta_{i} \epsilon_{i} q_{1}}{z_{i}-w_{1}}} \mathrm{e}^{\sqrt{2 \alpha^{\prime}} \frac{\theta_{i} \epsilon_{i} q_{2}}{z_{i}-w_{2}}}\right) \frac{\left(\epsilon_{q_{1}} \epsilon_{q_{2}}\right)}{\left(w_{1}-w_{2}\right)^{2}}
\end{aligned}
$$

Proceeding as in the previous section we get the integral in eq. (A.1) computed in eq. (A.25). Inserting it in the previous equation, we get

$$
H_{n}=-\frac{g_{p+1}^{2}}{2 q_{1} q_{2}}\left[\frac{k_{n}\left(q_{2}-q_{1}\right)+q_{1} q_{2}}{k_{n}\left(q_{1}+q_{2}\right)+q_{1} q_{2}}+\frac{k_{n-1}\left(q_{1}-q_{2}\right)+q_{1} q_{2}}{k_{n-1}\left(q_{1}+q_{2}\right)+q_{1} q_{2}}\right],
$$

that implies

$$
M_{2 s ; n g}=-\frac{g_{p+1}^{2}}{2 q_{1} q_{2}}\left[\frac{k_{n}\left(q_{2}-q_{1}\right)+q_{1} q_{2}}{k_{n}\left(q_{1}+q_{2}\right)+q_{1} q_{2}}+\frac{k_{n-1}\left(q_{1}-q_{2}\right)+q_{1} q_{2}}{k_{n-1}\left(q_{1}+q_{2}\right)+q_{1} q_{2}}\right] M_{n g} .
$$

\section{Double-soft behavior with $n+2$ scalars}

In this section we compute the double-soft behavior of two scalars in an amplitude with $n$ additional scalar particles instead of gluons. The set-up and the procedure is as in the section for gluons. The scattering amplitude is given by:

$$
\begin{aligned}
M_{2 s ; n s}= & \frac{\left(\sqrt{2 \alpha^{\prime}}\right)^{n}}{\alpha^{\prime}} g_{p+1}^{n} \int \frac{\prod_{i=1}^{n} d z_{i}}{d V_{a b c}} \prod_{i=1}^{n} d \theta_{i} \int_{0}^{z_{n-1}} d w_{1} \int_{0}^{w_{1}} d w_{2} \int d \phi_{1} d \phi_{2} \\
& \times\langle 0| \prod_{i=1}^{n-1} \mathrm{e}^{i\left(\theta_{i} \epsilon_{i} \partial_{z_{i}}+\sqrt{2 \alpha^{\prime}} k_{i}\right) X\left(z_{i}\right)} \\
& \quad \mathrm{e}^{i\left(\phi_{1} \epsilon_{q_{1}} \partial_{w_{1}}+\sqrt{2 \alpha^{\prime}} q_{1}\right) X\left(w_{1}\right)} \mathrm{e}^{i\left(\phi_{2} \epsilon_{q_{2}} \partial_{w_{2}}+\sqrt{2 \alpha^{\prime}} q_{2}\right) X\left(w_{2}\right)} \mathrm{e}^{i\left(\theta_{n} \epsilon_{n} \partial_{z_{n}}+\sqrt{2 \alpha^{\prime}} k_{n}\right) X\left(z_{n}\right)}|0\rangle .
\end{aligned}
$$


Contracting the vertex operators of the states with momenta $q_{1}$ and $q_{2}$ and remembering that, in this case, all momenta are orthogonal to all polarizations, we get

$$
\begin{aligned}
M_{2 s ; n s}= & \frac{\left(\sqrt{2 \alpha^{\prime}}\right)^{n}}{\alpha^{\prime}} g_{p+1}^{n} \int \frac{\prod_{i=1}^{n} d z_{i}}{d V_{a b c}} \prod_{i=1}^{n} d \theta_{i}\left\langle 0\left|\prod_{i=1}^{n} \mathrm{e}^{i\left(\theta_{i} \epsilon \partial_{z_{i}}+\sqrt{2 \alpha^{\prime}} k_{i}\right) X\left(z_{i}\right)}\right| 0\right\rangle \\
& \times \int_{0}^{z_{n-1}} d w_{1} \int_{0}^{w_{1}} d w_{2} \prod_{i=1}^{n-1}\left(\left(z_{i}-w_{1}\right)^{2 \alpha^{\prime} k_{i} q_{1}}\left(z_{i}-w_{2}\right)^{2 \alpha^{\prime} k_{i} q_{2}}\right) \\
& \times\left(w_{1}-z_{n}\right)^{2 \alpha^{\prime} q_{1} k_{n}}\left(w_{2}-z_{n}\right)^{2 \alpha^{\prime} q_{2} k_{n}}\left(w_{1}-w_{2}\right)^{2 \alpha^{\prime} q_{1} q_{2}} \\
& \times \int d \phi_{1} d \phi_{2} \mathrm{e}^{-\phi_{1} \phi_{2}} \frac{\left(\epsilon_{q_{1}} \epsilon q_{2}\right)}{\left(w_{1}-w_{2}\right)^{2}} \mathrm{e}^{\phi_{1} \sum_{i=1}^{n} \frac{\theta_{i}\left(\epsilon_{i} \epsilon q_{1}\right)}{\left(z_{i}-w_{1}\right)^{2}}} \mathrm{e}^{\phi_{2} \sum_{i=1}^{n} \frac{\theta_{i}\left(\epsilon_{i} \epsilon q_{2}\right)}{\left(z_{i}-w_{2}\right)^{2}}} .
\end{aligned}
$$

We can now integrate over $\phi_{1}$ and $\phi_{2}$

$$
\begin{aligned}
M_{2 s ; n s}= & g_{p+1}^{n-2} \frac{\left(\sqrt{2 \alpha^{\prime}}\right)^{n-2}}{\alpha^{\prime}} \int \frac{\prod_{i=1}^{n} d z_{i}}{d V_{a b c}} \prod_{i=1}^{n} d \theta_{i}\left\langle 0\left|\prod_{i=1}^{n} \mathrm{e}^{i\left(\theta_{i} \epsilon \partial_{z_{i}}+\sqrt{2 \alpha^{\prime}} k_{i}\right) X\left(z_{i}\right)}\right| 0\right\rangle \\
& \times 2 \alpha^{\prime} g_{p+1}^{2} \int_{0}^{z_{n-1}} d w_{1} \int_{0}^{w_{1}} d w_{2} \prod_{i=1}^{n}\left(\left(z_{i}-w_{1}\right)^{2 \alpha^{\prime} k_{i} q_{1}}\left(z_{i}-w_{2}\right)^{\left.2 \alpha^{\prime} k_{i} q_{2}\right)}\right. \\
& \times\left(w_{1}-w_{2}\right)^{2 \alpha^{\prime} q_{1} q_{2}}\left[\frac{\left(\epsilon_{q_{1}} \epsilon_{q_{2}}\right)}{\left(w_{1}-w_{2}\right)^{2}}+\sum_{i, j=1}^{n} \frac{\theta_{i} \theta_{j}\left(\epsilon_{q_{1}} \epsilon_{i}\right)\left(\epsilon_{q_{2}} \epsilon_{j}\right)}{\left(z_{i}-w_{1}\right)^{2}\left(z_{j}-w_{2}\right)^{2}}\right] \equiv M_{n s} * S_{n},
\end{aligned}
$$

where

$$
M_{n s}=g_{p+1}^{n-2} \frac{\left(\sqrt{2 \alpha^{\prime}}\right)^{n-2}}{\alpha^{\prime}} \int \frac{\prod_{i=1}^{n} d z_{i}}{d V_{a b c}} \prod_{i=1}^{n} d \theta_{i}\left\langle 0\left|\prod_{i=1}^{n} \mathrm{e}^{i\left(\theta_{i} \epsilon_{i} \partial_{z_{i}}+\sqrt{2 \alpha^{\prime}} k_{i}\right) X\left(z_{i}\right)}\right| 0\right\rangle,
$$

and

$$
\begin{aligned}
S_{n}= & 2 \alpha^{\prime} g_{p+1}^{2} \int_{0}^{z_{n-1}} d w_{1} \int_{0}^{w_{1}} d w_{2} \prod_{i=1}^{n}\left(\left(z_{i}-w_{1}\right)^{2 \alpha^{\prime} k_{i} q_{1}}\left(z_{i}-w_{2}\right)^{2 \alpha^{\prime} k_{i} q_{2}}\right) \\
& \times\left(w_{1}-w_{2}\right)^{2 \alpha^{\prime} q_{1} q_{2}}\left[\frac{\left(\epsilon_{q_{1}} \epsilon_{q_{2}}\right)}{\left(w_{1}-w_{2}\right)^{2}}+\sum_{i, j=1}^{n} \frac{\theta_{i} \theta_{j}\left(\epsilon_{q_{1}} \epsilon_{i}\right)\left(\epsilon_{q_{2}} \epsilon_{j}\right)}{\left(z_{i}-w_{1}\right)^{2}\left(z_{j}-w_{2}\right)^{2}}\right] .
\end{aligned}
$$

Similar to the case of gluons, we keep only the leading terms in the double-soft limit. It is also convenient to introduce the new variables $w_{1}=z_{n-1} \hat{w}_{1}$ and $w_{2}=z_{n-1} \hat{w}_{2}$. Then, the previous expression becomes

$$
\begin{aligned}
S_{n}= & 2 \alpha^{\prime} g_{p+1}^{2} z_{n-1}^{2 \alpha^{\prime}\left[q_{1} q_{2}+\left(k_{n}+k_{n-1}\right)\left(q_{1}+q_{2}\right)\right]} \int_{0}^{1} d \hat{w}_{1} \int_{0}^{\hat{w}_{1}} d \hat{w}_{2} \\
& \times\left(1-\hat{w}_{1}\right)^{2 \alpha^{\prime} k_{n-1} q_{1}}\left(1-\hat{w}_{2}\right)^{2 \alpha^{\prime} k_{n-1} q_{2}} \hat{w}_{1}^{2 \alpha^{\prime} k_{n} q_{1}} \hat{w}_{2}^{2 \alpha^{\prime} k_{n} q_{2}}\left(\hat{w}_{1}-\hat{w}_{2}\right)^{2 \alpha^{\prime} q_{1} q_{2}} \\
& \times\left[\frac{\left(\epsilon_{q_{1}} \epsilon_{q_{2}}\right)}{\left(\hat{w}_{1}-\hat{w}_{2}\right)^{2}}+z_{n-1}^{2} \sum_{i, j=1}^{n} \frac{\theta_{i} \theta_{j}\left(\epsilon_{q_{1}} \epsilon_{i}\right)\left(\epsilon_{q_{2}} \epsilon_{j}\right)}{\left(z_{i}-z_{n-1} \hat{w}_{1}\right)^{2}\left(z_{j}-z_{n-1} \hat{w}_{2}\right)^{2}}\right] .
\end{aligned}
$$

Also in this case we have two kinds of terms. The first one is without any dependence on the variables $\theta_{i}$ and the convolution multiplies the amplitude with $n$ scalars, while the second 
term contains terms with $\theta_{i}$ that modify the structure of $M_{n s}$. Also in this case it can be shown that only the first term contributes to the leading double-soft limit. Therefore, here we concentrate on the first term taking the term $z_{n-1}^{2 \alpha^{\prime}\left[q_{1} q_{2}+\left(k_{n}+k_{n-1}\right)\left(q_{1}+q_{2}\right)\right]}=1$. We get the integral in eq. (A.1) that has been computed in eq. (A.25) and therefore for the first term in the squared bracket in eq. (4.6), one gets:

$$
S_{n}^{(1)}=-\frac{g_{p+1}^{2}}{2 q_{1} q_{2}}\left[\frac{k_{n}\left(q_{2}-q_{1}\right)+q_{1} q_{2}}{k_{n}\left(q_{1}+q_{2}\right)+q_{1} q_{2}}+\frac{k_{n-1}\left(q_{1}-q_{2}\right)+q_{1} q_{2}}{k_{n-1}\left(q_{1}+q_{2}\right)+q_{1} q_{2}}\right] .
$$

This means that the double-soft behavior in an amplitude with $n+2$ scalars is given by

$$
M_{2 s ; n s}=-\frac{g_{p+1}^{2}}{2 q_{1} q_{2}}\left[\frac{k_{n}\left(q_{2}-q_{1}\right)+q_{1} q_{2}}{k_{n}\left(q_{1}+q_{2}\right)+q_{1} q_{2}}+\frac{k_{n-1}\left(q_{1}-q_{2}\right)+q_{1} q_{2}}{k_{n-1}\left(q_{1}+q_{2}\right)+q_{1} q_{2}}\right] M_{n s} .
$$

\section{Conclusions}

In this paper we have computed the leading double-soft behavior of the scattering amplitudes with gluons and scalars living in the world-volume of a $\mathrm{D} p$-brane of the bosonic string. The corresponding field-theory results can be obtained by just sending $\alpha^{\prime}$ to zero. Our results are valid in any number of space-time dimensions and for an arbitrary gauge choice. We have also for the first time considered and provided the results for the doublesoft limit of two gluons that are not contiguous, but separated by a hard gluon in the color-ordered amplitude.

\section{Acknowledgments}

We thank Massimo Bianchi, Andrea Guerrieri and Congkao Wen for discussions.

\section{A Computation of various integrals to the leading order in the double- soft limit}

In this appendix we compute the contribution of various integrals in the double-soft limit.

Let us start considering the first integral appearing in eq. (2.8):

$$
\begin{aligned}
I\left(k_{2}, q_{1} ; k_{3}, q_{2}\right) \equiv & \int_{0}^{1} d w_{1} \int_{0}^{w_{1}} d w_{2} w_{1}^{2 \alpha^{\prime} q_{1} k_{3}} w_{2}^{2 \alpha^{\prime} q_{2} k_{3}}\left(1-w_{1}\right)^{2 \alpha^{\prime} k_{2} q_{1}}\left(1-w_{2}\right)^{2 \alpha^{\prime} k_{2} q_{2}} \\
& \times\left(w_{1}-w_{2}\right)^{2 \alpha^{\prime} q_{1} q_{2}-2}
\end{aligned}
$$

and let us show that it is symmetric under the simultaneous exchange of $k_{2} \leftrightarrow k_{3}$ and $q_{1} \leftrightarrow q_{2}$. It is convenient to introduce the variables:

$$
x_{4} \equiv w_{2} ; \quad x_{3} \equiv 1-w_{1}
$$

The integral becomes:

$$
\begin{aligned}
I\left(k_{2}, q_{1} ; k_{3}, q_{2}\right)= & \int_{0}^{1} d x_{3}\left(1-x_{3}\right)^{2 \alpha^{\prime} q_{1} k_{3}} x_{3}^{2 \alpha^{\prime} q_{1} k_{2}} \int_{0}^{1-x_{3}} d x_{4} x_{4}^{2 \alpha^{\prime} q_{2} k_{3}}\left(1-x_{4}\right)^{2 \alpha^{\prime} k_{2} q_{2}} \\
& \times\left(1-x_{3}-x_{4}\right)^{2 \alpha^{\prime} q_{1} q_{2}-2}
\end{aligned}
$$


It is possible to give an equivalent expression of the integral (A.1):

$$
\begin{aligned}
I\left(k_{2}, q_{1} ; k_{3}, q_{2}\right)= & \int_{0}^{1} d w_{2} w_{2}^{2 \alpha^{\prime} q_{2} k_{3}}\left(1-w_{2}\right)^{2 \alpha^{\prime} k_{2} q_{2}} \int_{w_{2}}^{1} d w_{1} w_{1}^{2 \alpha^{\prime} q_{1} k_{3}}\left(1-w_{1}\right)^{2 \alpha^{\prime} q_{1} k_{2}} \\
& \times\left(w_{1}-w_{2}\right)^{2 \alpha^{\prime} q_{1} q_{2}-2}
\end{aligned}
$$

Introducing again the variables in eq. (A.2), we get

$$
\begin{aligned}
I\left(k_{2}, q_{1} ; k_{3}, q_{2}\right)= & \int_{0}^{1} d x_{4} x_{4}^{2 \alpha^{\prime} q_{2} k_{3}}\left(1-x_{4}\right)^{2 \alpha^{\prime} k_{2} q_{2}} \int_{0}^{1-x_{4}} d x_{3}\left(1-x_{3}\right)^{2 \alpha^{\prime} q_{1} k_{3}} x_{3}^{2 \alpha^{\prime} q_{1} k_{2}} \\
& \times\left(1-x_{3}-x_{4}\right)^{2 \alpha^{\prime} q_{1} q_{2}-2}
\end{aligned}
$$

Both representations can be collected in the formula:

$$
\begin{aligned}
I\left(k_{2}, q_{1} ; k_{3}, q_{2}\right)= & \int_{0}^{1} \prod_{i=1}^{3} d x_{i} \delta\left(1-x_{2}-x_{3}-x_{4}\right) x_{4}^{2 \alpha^{\prime} q_{2} k_{3}}\left(1-x_{4}\right)^{2 \alpha^{\prime} k_{2} q_{2}}\left(1-x_{3}\right)^{2 \alpha^{\prime} q_{1} k_{3}} \\
& \times x_{3}^{2 \alpha^{\prime} q_{1} k_{2}} x_{2}^{2 \alpha^{\prime} q_{1} q_{2}-2}
\end{aligned}
$$

Let us now keep the variable $x_{2}$ and solve the delta function by eliminating either $x_{3}$ or $x_{4}$. By eliminating $x_{3}=1-x_{2}-x_{4}$ one gets:

$$
\begin{aligned}
I\left(k_{2}, q_{1} ; k_{3}, q_{2}\right)= & \int_{0}^{1} d x_{2} \int_{0}^{1-x_{2}} d x_{4} x_{4}^{2 \alpha^{\prime} q_{2} k_{3}}\left(1-x_{4}\right)^{2 \alpha^{\prime} k_{2} q_{2}} \\
& \times\left(x_{2}+x_{4}\right)^{2 \alpha^{\prime} q_{1} k_{3}}\left(1-x_{2}-x_{4}\right)^{2 \alpha^{\prime} q_{1} k_{2}} x_{2}^{2 \alpha^{\prime} q_{1} q_{2}-2}
\end{aligned}
$$

Introducing the variables $x_{2}=\rho \omega ; x_{4}=\rho(1-\omega)$ one gets:

$$
\begin{aligned}
I\left(k_{2}, q_{1} ; k_{3}, q_{2}\right)= & \int_{0}^{1} d \rho \rho \int_{0}^{1} d \omega \rho^{2 \alpha^{\prime} q_{2} k_{3}}(1-\omega)^{2 \alpha^{\prime} q_{2} k_{3}}(1-\rho(1-\omega))^{2 \alpha^{\prime} k_{2} q_{2}} \\
& \times \rho^{2 \alpha^{\prime} q_{1} k_{3}}(1-\rho)^{2 \alpha^{\prime} q_{1} k_{2}} \rho^{2 \alpha^{\prime} q_{1} q_{2}-2} \omega^{2 \alpha^{\prime} q_{1} q_{2}-2}
\end{aligned}
$$

By changing variable $t=1-\omega$, one gets:

$$
\begin{aligned}
I\left(k_{2}, q_{1} ; k_{3}, q_{2}\right)= & \int_{0}^{1} d \rho \rho^{2 \alpha^{\prime}\left(q_{2}+q_{1}\right) \cdot k_{3}+2 \alpha^{\prime} q_{1} q_{2}-1}(1-\rho)^{2 \alpha^{\prime} q_{1} k_{2}} \int_{0}^{1} d t t^{2 \alpha^{\prime} q_{2} k_{3}} \\
& \times(1-\rho t)^{2 \alpha^{\prime} k_{2} q_{2}}(1-t)^{2 \alpha^{\prime} q_{1} q_{2}-2}
\end{aligned}
$$

Let us now eliminate instead $x_{4}=1-x_{2}-x_{3}$. One gets:

$$
\begin{aligned}
I\left(k_{2}, q_{1} ; k_{3}, q_{2}\right)= & \int_{0}^{1} d x_{2} \int_{0}^{1-x_{2}} d x_{3}\left(1-x_{2}-x_{3}\right)^{2 \alpha^{\prime} q_{2} k_{3}}\left(x_{2}+x_{3}\right)^{2 \alpha^{\prime} k_{2} q_{2}}\left(1-x_{3}\right)^{2 \alpha^{\prime} q_{1} k_{3}} \\
& \times x_{3}^{2 \alpha^{\prime} q_{1} k_{2}} x_{2}^{2 \alpha^{\prime} q_{1} q_{2}-2}
\end{aligned}
$$

By defining again $x_{2}=\rho \omega ; x_{3}=\rho(1-\omega)$ one gets:

$$
\begin{aligned}
I\left(k_{2}, q_{1} ; k_{3}, q_{2}\right)= & \int_{0}^{1} d \rho \rho \int_{0}^{1} d \omega(1-\rho)^{2 \alpha^{\prime} q_{2} k_{3}} \rho^{2 \alpha^{\prime} k_{2} q_{2}}(1-\rho(1-\omega))^{2 \alpha^{\prime} q_{1} k_{3}} \rho^{2 \alpha^{\prime} q_{1} k_{2}} \\
& \times(1-\omega)^{2 \alpha^{\prime} q_{1} k_{2}} \rho^{2 \alpha^{\prime} q_{1} q_{2}-2} \omega^{2 \alpha^{\prime} q_{1} q_{2}-2}
\end{aligned}
$$


This equation is obtained from eq. (A.8) by changing $q_{1} \leftrightarrow q_{2}$ and $k_{2} \leftrightarrow k_{3}$ and we get

$$
I\left(k_{2}, q_{1} ; k_{3}, q_{2}\right)=I\left(k_{3}, q_{2} ; k_{2}, q_{1}\right)
$$

Let us now compute it in the double-soft limit. Let us first change variables $w_{1}=z$ and $w_{2}=x w_{1}$ in eq. (A.1) getting

$$
I=\int_{0}^{1} d z z^{2 \alpha^{\prime}\left(k_{3}\left(q_{1}+q_{2}\right)+q_{1} q_{2}\right)-1}(1-z)^{2 \alpha^{\prime} k_{2} q_{1}} \int_{0}^{1} d x x^{2 \alpha^{\prime} q_{2} k_{3}}(1-x)^{2 \alpha^{\prime} q_{1} q_{2}-2}(1-x z)^{2 \alpha^{\prime} k_{2} q_{2}}
$$

Then, we use the following equation

$$
\int_{0}^{1} d t t^{b-1}(1-t)^{c-b-1}(1-t y)^{-a}=\frac{\Gamma(b) \Gamma(c-b)}{\Gamma(c)}{ }_{2} F_{1}(a, b ; c ; y)
$$

to rewrite eq. (A.13) as follows

$$
I=\frac{\Gamma(b) \Gamma(c-b)}{\Gamma(c)} \int_{0}^{1} d z z^{2 \alpha^{\prime}\left(k_{3}\left(q_{1}+q_{2}\right)+q_{1} q_{2}\right)-1}(1-z)^{2 \alpha^{\prime} k_{2} q_{1}}{ }_{2} F_{1}(a, b ; c ; z)
$$

with

$$
\begin{aligned}
b & =1+2 \alpha^{\prime} q_{2} k_{3} ; & a & =-2 \alpha^{\prime} k_{2} q_{2} \\
c-b & =2 \alpha^{\prime} q_{1} q_{2}-1 ; & c & =2 \alpha^{\prime}\left(q_{1}+k_{3}\right) q_{2}
\end{aligned}
$$

Then we can use

$$
{ }_{2} F_{1}(a, b ; c z)=(1-z)^{-a}{ }_{2} F_{1}\left(c-b, a ; c ; \frac{z}{1-z}\right)
$$

to get

$$
\begin{aligned}
I= & \frac{\Gamma(b) \Gamma(c-b)}{\Gamma(a) \Gamma(c-a)} \int_{0}^{1} d z z^{2 \alpha^{\prime}\left(k_{3}\left(q_{1}+q_{2}\right)+q_{1} q_{2}\right)-1}(1-z)^{2 \alpha^{\prime} k_{2}\left(q_{1}+q_{2}\right)} \\
& \times \int_{0}^{1} d x x^{a-1}(1-x)^{c-a-1}\left(1-\frac{z x}{z-1}\right)^{b-c} \\
= & \frac{\Gamma(b) \Gamma(c-b)}{\Gamma(a) \Gamma(c-a)} \int_{0}^{1} d z z^{2 \alpha^{\prime}\left(k_{3}\left(q_{1}+q_{2}\right)+q_{1} q_{2}\right)-1}(1-z)^{2 \alpha^{\prime} k_{2}\left(q_{1}+q_{2}\right)} \\
& \times \int_{0}^{1} d t(1-t)^{a-1} t^{c-a-1}\left(\frac{1-z t}{1-z}\right)^{b-c}
\end{aligned}
$$

It is equal to

$$
\begin{aligned}
I= & \frac{\Gamma(b) \Gamma(c-b)}{\Gamma(a) \Gamma(c-a)} \int_{0}^{1} d z z^{2 \alpha^{\prime}\left(k_{3}\left(q_{1}+q_{2}\right)+q_{1} q_{2}\right)-1}(1-z)^{2 \alpha^{\prime}\left(k_{2}\left(q_{1}+q_{2}\right)+q_{1} q_{2}\right)-1} \\
& \times \int_{0}^{1} d t(1-t)^{-2 \alpha^{\prime} k_{2} q_{2}-1} t^{2 \alpha^{\prime} q_{2}\left(k_{2}+k_{3}+q_{1}\right)-1}(1-z t)^{1-2 \alpha^{\prime} q_{1} q_{2}}
\end{aligned}
$$

Introducing, for the sake of simplicity, the momentum $k_{1}=-k_{2}-k_{3}-q_{1}-q_{2}$, yields

$$
\begin{aligned}
I= & \frac{\Gamma(b) \Gamma\left(2 \alpha^{\prime} q_{1} q_{2}-1\right)}{\Gamma\left(-2 \alpha^{\prime} k_{2} q_{2}\right) \Gamma\left(-2 \alpha^{\prime} q_{2} k_{1}\right)} \int_{0}^{1} d z z^{2 \alpha^{\prime}\left(k_{3}\left(q_{1}+q_{2}\right)+q_{1} q_{2}\right)-1}(1-z)^{2 \alpha^{\prime}\left(k_{2}\left(q_{1}+q_{2}\right)+q_{1} q_{2}\right)-1} \\
& \times \int_{0}^{1} d t(1-t)^{-2 \alpha^{\prime} k_{2} q_{2}-1} t^{-2 \alpha^{\prime} q_{2} k_{1}-1}(1-z t)^{1-2 \alpha^{\prime} q_{1} q_{2}}
\end{aligned}
$$


Keeping only the term with 1 in the exponent of the last term, we can easily compute the two integrals. One gets:

$$
\begin{aligned}
I= & \frac{\Gamma(b) \Gamma\left(2 \alpha^{\prime} q_{1} q_{2}-1\right)}{\Gamma\left(-2 \alpha^{\prime} k_{2} q_{2}\right) \Gamma\left(-2 \alpha^{\prime} q_{2} k_{1}\right)}\left[\frac{\Gamma\left(2 \alpha^{\prime}\left(k_{3}\left(q_{1}+q_{2}\right)+q_{1} q_{2}\right)\right) \Gamma\left(2 \alpha^{\prime}\left(k_{2}\left(q_{1}+q_{2}\right)+q_{1} q_{2}\right)\right)}{\Gamma\left(2 \alpha^{\prime}\left(\left(k_{3}+k_{2}\right)\left(q_{1}+q_{2}\right)+2 q_{1} q_{2}\right)\right)}\right. \\
& \times \frac{\Gamma\left(-2 \alpha^{\prime} k_{2} q_{2}\right) \Gamma\left(-2 \alpha^{\prime} q_{2} k_{1}\right)}{\Gamma\left(-2 \alpha^{\prime} q_{2}\left(k_{1}+k_{2}\right)\right)}-\frac{\Gamma\left(2 \alpha^{\prime}\left(k_{3}\left(q_{1}+q_{2}\right)+q_{1} q_{2}\right)+1\right) \Gamma\left(2 \alpha^{\prime}\left(k_{2}\left(q_{1}+q_{2}\right)+q_{1} q_{2}\right)\right)}{\Gamma\left(2 \alpha^{\prime}\left(\left(k_{3}+k_{2}\right)\left(q_{1}+q_{2}\right)+2 q_{1} q_{2}\right)+1\right)} \\
& \left.\times \frac{\Gamma\left(-2 \alpha^{\prime} k_{2} q_{2}\right) \Gamma\left(1-2 \alpha^{\prime} q_{2} k_{1}\right)}{\Gamma\left(1-2 \alpha^{\prime} q_{2}\left(k_{1}+k_{2}\right)\right)}\right]
\end{aligned}
$$

It can be rewritten as follows

$$
\begin{aligned}
I= & \frac{\Gamma(b) \Gamma\left(2 \alpha^{\prime} q_{1} q_{2}+1\right)}{\left(2 \alpha^{\prime} q_{1} q_{2}\right)\left(2 \alpha^{\prime} q_{1} q_{2}-1\right) \Gamma\left(-2 \alpha^{\prime} q_{2} k_{1}\right)} \frac{\Gamma\left(1-2 \alpha^{\prime} q_{2} k_{1}\right)}{\Gamma\left(1-2 \alpha^{\prime} q_{2}\left(k_{1}+k_{2}\right)\right)} \\
& \times \frac{\Gamma\left(1+2 \alpha^{\prime}\left(k_{3}\left(q_{1}+q_{2}\right)+q_{1} q_{2}\right)\right) \Gamma\left(1+2 \alpha^{\prime}\left(k_{2}\left(q_{1}+q_{2}\right)+q_{1} q_{2}\right)\right)}{\Gamma\left(1+2 \alpha^{\prime}\left(\left(k_{3}+k_{2}\right)\left(q_{1}+q_{2}\right)+2 q_{1} q_{2}\right)\right)} \\
& \times\left[\frac{q_{2}\left(k_{1}+k_{2}\right)}{2 \alpha^{\prime} q_{2} k_{1}}\left(\frac{1}{\left(k_{3}\left(q_{1}+q_{2}\right)+q_{1} q_{2}\right)}+\frac{1}{\left(k_{2}\left(q_{1}+q_{2}\right)+q_{1} q_{2}\right)}\right)\right. \\
& \left.-\frac{1}{2 \alpha^{\prime}\left(k_{2}\left(q_{1}+q_{2}\right)+q_{1} q_{2}\right)}\right]
\end{aligned}
$$

that is equal to

$$
\begin{aligned}
I= & \frac{\Gamma(b)(-1) \Gamma\left(2 \alpha^{\prime} q_{1} q_{2}+1\right)}{\left(2 \alpha^{\prime} q_{1} q_{2}\right)\left(2 \alpha^{\prime} q_{1} q_{2}-1\right) \Gamma\left(1-2 \alpha^{\prime} q_{2} k_{1}\right)} \frac{\Gamma\left(1-2 \alpha^{\prime} q_{2} k_{1}\right)}{\Gamma\left(1-2 \alpha^{\prime} q_{2}\left(k_{1}+k_{2}\right)\right)} \\
& \times \frac{\Gamma\left(1+2 \alpha^{\prime}\left(k_{3}\left(q_{1}+q_{2}\right)+q_{1} q_{2}\right)\right) \Gamma\left(1+2 \alpha^{\prime}\left(k_{2}\left(q_{1}+q_{2}\right)+q_{1} q_{2}\right)\right)}{\Gamma\left(1+2 \alpha^{\prime}\left(\left(k_{3}+k_{2}\right)\left(q_{1}+q_{2}\right)+2 q_{1} q_{2}\right)\right)} \\
& \times\left[\frac{q_{2}\left(k_{1}+k_{2}\right)}{\left(k_{3}\left(q_{1}+q_{2}\right)+q_{1} q_{2}\right)}+\frac{k_{2} q_{2}}{k_{2}\left(q_{1}+q_{2}\right)+q_{1} q_{2}}\right]
\end{aligned}
$$

In the double-soft limit one gets:

$$
I^{f t}=\frac{1}{2 \alpha^{\prime} q_{1} q_{2}}\left[-\frac{q_{2}\left(k_{3}+q_{1}\right)}{k_{3}\left(q_{1}+q_{2}\right)+q_{1} q_{2}}+\frac{k_{2} q_{2}}{k_{2}\left(q_{1}+q_{2}\right)+q_{1} q_{2}}\right]
$$

Because of the symmetry in eq. (A.12), we must symmetrize the previous expression getting:

$$
I^{f t}=-\frac{1}{4 \alpha^{\prime} q_{1} q_{2}}\left[\frac{k_{3}\left(q_{2}-q_{1}\right)+q_{1} q_{2}}{k_{3}\left(q_{1}+q_{2}\right)+q_{1} q_{2}}+\frac{k_{2}\left(q_{1}-q_{2}\right)+q_{1} q_{2}}{k_{2}\left(q_{1}+q_{2}\right)+q_{1} q_{2}}\right]
$$

In the second part of this section we compute the remaining nine integrals in eq. (2.7) in the double-soft limit ( $q_{1}$ and $q_{2}$ going simultaneously to zero).

The term with the denominator $\frac{1}{w_{1}\left(w_{1}-w_{2}\right)}$ can be written as follows after having taken $w_{2}=w_{1} x:$

$$
\begin{aligned}
I_{w_{1} ; w_{1}-w_{2}} \equiv & \int_{0}^{1} d w_{1} \int_{0}^{1} d x w_{1}^{2 \alpha^{\prime}\left(k_{3}\left(q_{1}+q_{2}\right)+q_{1} q_{2}\right)-1}(1-x)^{2 \alpha^{\prime} q_{1} q_{2}-1}\left(1-w_{1}\right)^{2 \alpha^{\prime} k_{2} q_{1}} \\
& \times\left(1-w_{1} x\right)^{2 \alpha^{\prime} k_{2} q_{2}} w_{1}^{2 \alpha^{\prime} q_{1} k_{3}} x^{2 \alpha^{\prime} q_{2} k_{3}} \\
& \sim \frac{1}{\left(2 \alpha^{\prime} q_{1} q_{2}\right) 2 \alpha^{\prime}\left(k_{3}\left(q_{1}+q_{2}\right)+q_{1} q_{2}\right)}
\end{aligned}
$$


The term with the denominator $\frac{1}{w_{2}\left(w_{1}-w_{2}\right)}$ can be written as follows after having taken $w_{2}=w_{1} x$ :

$$
\begin{aligned}
I_{w_{2} ; w_{1}-w_{2}} \equiv & \int_{0}^{1} d w_{1} \int_{0}^{1} d x w_{1}^{2 \alpha^{\prime}\left(\left(q_{1}+q_{2}\right) k_{3}+q_{1} q_{2}\right)-1}(1-x)^{2 \alpha^{\prime} q_{1} q_{2}-1} \\
& \times\left(1-w_{1} x\right)^{2 \alpha^{\prime} k_{2} q_{2}}\left(1-w_{1}\right)^{2 \alpha^{\prime} k_{2} q_{1}} x^{2 \alpha^{\prime} q_{2} k_{3}-1} \\
\sim & \frac{1}{2 \alpha^{\prime}\left(\left(q_{1}+q_{2}\right) k_{3}+q_{1} q_{2}\right)} \frac{\Gamma\left(2 \alpha^{\prime} k_{3} q_{2}\right) \Gamma\left(2 \alpha^{\prime} q_{1} q_{2}\right)}{\Gamma\left(2 \alpha^{\prime}\left(q_{1} q_{2}+k_{3} q_{2}\right)\right)} \\
\sim & \frac{1}{2 \alpha^{\prime}\left(\left(q_{1}+q_{2}\right) k_{3}+q_{1} q_{2}\right)} \frac{2 \alpha^{\prime}\left(q_{1} q_{2}+k_{3} q_{2}\right)}{\left(2 \alpha^{\prime} q_{1} q_{2}\right)\left(2 \alpha^{\prime} k_{3} q_{2}\right)} \\
= & \frac{1}{\left(2 \alpha^{\prime} q_{1} q_{2}\right) 2 \alpha^{\prime}\left(k_{3}\left(q_{1}+q_{2}\right)+q_{1} q_{2}\right)}
\end{aligned}
$$

The term with the denominator $\frac{1}{\left(1-w_{2}\right)\left(w_{1}-w_{2}\right)}$ can be written as follows:

$$
\begin{aligned}
I_{1-w_{2} ; w_{1}-w_{2}} \equiv & \int_{0}^{1} d w_{2} \int_{w_{2}}^{1} d w_{2}\left(w_{1}-w_{2}\right)^{2 \alpha^{\prime} q_{1} q_{2}-1}\left(1-w_{1}\right)^{2 \alpha^{\prime} k_{2} q_{1}} \\
& \times\left(1-w_{2}\right)^{2 \alpha^{\prime} k_{2} q_{2}-1} w_{1}^{2 \alpha^{\prime} q_{1} k_{3}} w_{2}^{2 \alpha^{\prime} q_{2} k_{3}}
\end{aligned}
$$

Introducing the new variables $w_{1}=1-x_{1}$ and $w_{2}=1-x_{2}$, one gets

$$
\begin{aligned}
I_{1-w_{2} ; w_{1}-w_{2}} \equiv & \int_{0}^{1} d x_{2} \int_{0}^{x_{2}} d x_{1}\left(x_{2}-x_{1}\right)^{2 \alpha^{\prime} q_{1} q_{2}-1} x_{1}^{2 \alpha^{\prime} k_{2} q_{1}} x_{2}^{2 \alpha^{\prime} k_{2} q_{2}-1} \\
& \times\left(1-x_{1}\right)^{2 \alpha^{\prime} q_{1} k_{3}}\left(1-x_{2}\right)^{2 \alpha^{\prime} q_{2} k_{3}}
\end{aligned}
$$

By introducing the variable $x_{1}=x_{2} x$ we get

$$
\begin{aligned}
I_{1-w_{2} ; w_{1}-w_{2}} \equiv & \int_{0}^{1} d x_{2} \int_{0}^{1} d x x_{2}^{2 \alpha^{\prime}\left(q_{1} q_{2}+k_{2}\left(q_{1}+q_{2}\right)\right)-1}(1-x)^{2 \alpha^{\prime} q_{1} q_{2}-1} x^{2 \alpha^{\prime} k_{2} q_{1}} \\
& \times x_{2}^{2 \alpha^{\prime} k_{2} q_{2}-1}\left(1-x_{2} x\right)^{2 \alpha^{\prime} q_{1} k_{3}}\left(1-x_{2}\right)^{2 \alpha^{\prime} q_{2} k_{3}} \\
\sim & \frac{1}{\left(2 \alpha^{\prime} q_{1} q_{2}\right) 2 \alpha^{\prime}\left(q_{1} q_{2}+k_{2}\left(q_{1}+q_{2}\right)\right)}
\end{aligned}
$$

The term with the denominator $\frac{1}{\left(1-w_{1}\right)\left(w_{1}-w_{2}\right)}$ can be written as:

$$
\begin{aligned}
I_{1-w_{1} ; w_{1}-w_{2}} \equiv & \int_{0}^{1} d w_{2} \int_{w_{2}}^{1} d w_{1}\left(w_{1}-w_{2}\right)^{2 \alpha^{\prime} q_{1} q_{2}-1}\left(1-w_{1}\right)^{2 \alpha^{\prime} k_{2} q_{1}-1} \\
& \times\left(1-w_{2}\right)^{2 \alpha^{\prime} k_{2} q_{2}} w_{1}^{2 \alpha^{\prime} q_{1} k_{3}} w_{2}^{2 \alpha^{\prime} q_{2} k_{3}}
\end{aligned}
$$

Introducing the variables $w_{1}=1-x_{1}$ and $w_{2}=1-x_{2}$, one gets:

$$
\begin{aligned}
I_{1-w_{1} ; w_{1}-w_{2}} \equiv & \int_{0}^{1} d x_{2} \int_{0}^{x_{2}} d x_{1}\left(x_{2}-x_{1}\right)^{2 \alpha^{\prime} q_{1} q_{2}-1} x_{1}^{2 \alpha^{\prime} k_{2} q_{1}-1} x_{2}^{2 \alpha^{\prime} k_{2} q_{2}} \\
& \times\left(1-x_{1}\right)^{2 \alpha^{\prime} q_{1} k_{3}}\left(1-x_{2}\right)^{2 \alpha^{\prime} q_{2} k_{3}}
\end{aligned}
$$


Changing variable $x_{1}=x_{2} x$, we get

$$
\begin{aligned}
I_{1-w_{1} ; w_{1}-w_{2}} \equiv & \int_{0}^{1} d x_{2} \int_{0}^{1} d x x_{2}^{2 \alpha^{\prime}\left(k_{2}\left(q_{1}+q_{2}\right)+q_{1} q_{2}\right)-1}(1-x)^{2 \alpha^{\prime} q_{1} q_{2}-1} \\
& \times x^{2 \alpha^{\prime} k_{2} q_{1}-1}\left(1-x_{2} x\right)^{2 \alpha^{\prime} q_{1} k_{3}}\left(1-x_{2}\right)^{2 \alpha^{\prime} q_{2} k_{3}} \\
& \sim \frac{1}{2 \alpha^{\prime}\left(k_{2}\left(q_{1}+q_{2}\right)+q_{1} q_{2}\right)} \frac{\Gamma\left(2 \alpha^{\prime} q_{1} q_{2}\right) \Gamma\left(2 \alpha^{\prime} k_{2} q_{1}\right)}{\Gamma\left(2 \alpha^{\prime} q_{1}\left(q_{2}+k_{2}\right)\right)} \\
& \sim \frac{1}{2 \alpha^{\prime}\left(k_{2}\left(q_{1}+q_{2}\right)+q_{1} q_{2}\right)} \frac{2 \alpha^{\prime}\left(k_{2}\left(q_{1}+q_{2}\right)\right)}{\left(2 \alpha^{\prime} q_{1} q_{2}\right)\left(2 \alpha^{\prime} k_{2} q_{1}\right)} \\
= & \frac{1}{2 \alpha^{\prime}\left(k_{2}\left(q_{1}+q_{2}\right)+q_{1} q_{2}\right)} \frac{1}{2 \alpha^{\prime} q_{1} q_{2}}
\end{aligned}
$$

After the change of variable $w_{2}=w_{1} x$, the term with the denominator $\frac{1}{w_{1} w_{2}}$ is given by:

$$
\begin{aligned}
I_{w_{1} ; w_{2}} \equiv & \int_{0}^{1} d w_{1} w_{1}^{2 \alpha^{\prime}\left(k_{3}\left(q_{1}+q_{2}\right)+q_{1} q_{2}\right)-1}\left(1-w_{1}\right)^{2 \alpha^{\prime} k_{2} q_{1}} \int_{0}^{1} d x x^{2 \alpha^{\prime} q_{2} k_{3}-1} \\
& \times(1-x)^{2 \alpha^{\prime} q_{1} q_{2}}\left(1-x w_{1}\right)^{2 \alpha^{\prime} k_{2} q_{2}} \\
& \sim \frac{1}{2 \alpha^{\prime}\left(k_{3}\left(q_{1}+q_{2}\right)+q_{1} q_{2}\right)\left(2 \alpha^{\prime} k_{3} q_{2}\right)}
\end{aligned}
$$

The term with the denominator $\frac{1}{\left(1-w_{1}\right)\left(1-w_{2}\right)}$ after the change of variables $w_{1}=1-x_{1}$ and $w_{2}=1-x_{2}$ is equal to:

$$
\begin{aligned}
I_{1-w_{1} ; 1-w_{2}} & \equiv \int_{0}^{1} d x_{1} x_{1}^{2 \alpha^{\prime} q_{1} k_{2}-1}\left(1-x_{1}\right)^{2 \alpha^{\prime} q_{1} k_{3}} \int_{x_{1}}^{1} d x_{2}\left(1-x_{2}\right)^{2 \alpha^{\prime} q_{2} k_{3}} x_{2}^{2 \alpha^{\prime} k_{2} q_{2}-1}\left(x_{2}-x_{1}\right)^{2 \alpha^{\prime} q_{2} q_{1}} \\
& =\int_{0}^{1} d x_{2} x_{2}^{2 \alpha^{\prime} k_{2} q_{2}-1}\left(1-x_{2}\right)^{2 \alpha^{\prime} q_{2} k_{3}} \int_{0}^{x_{2}} d x_{1} x_{1}^{2 \alpha^{\prime} q_{1} k_{2}-1}\left(1-x_{1}\right)^{2 \alpha^{\prime} q_{1} k_{3}}\left(x_{2}-x_{1}\right)^{2 \alpha^{\prime} q_{2} q_{1}}
\end{aligned}
$$

After changing variable $x_{1}=x_{2} x$ we get

$$
\begin{aligned}
I_{1-w_{1} ; 1-w_{2}} \equiv & \int_{0}^{1} d x_{2} x_{2}^{2 \alpha^{\prime}\left(k_{2}\left(q_{1}+q_{2}\right)+q_{1} q_{2}\right)-1}\left(1-x_{2}\right)^{2 \alpha^{\prime} q_{2} k_{3}} \int_{0}^{1} d x x^{2 \alpha^{\prime} q_{1} k_{2}-1} \\
& \times(1-x)^{2 \alpha^{\prime} q_{1} q_{2}}\left(1-x_{2} x\right)^{2 \alpha^{\prime} q_{1} k_{3}} \\
& \sim \frac{1}{2 \alpha^{\prime}\left(k_{2}\left(q_{1}+q_{2}\right)+q_{1} q_{2}\right)\left(2 \alpha^{\prime} q_{1} k_{2}\right)}
\end{aligned}
$$

It is obtained from the one in eq. (A.34) with the substitution $k_{3} \leftrightarrow k_{2}$ and $q_{1} \leftrightarrow q_{2}$. 
The term with the factor $\left(1-w_{1}\right) w_{2}$ in the denominator is given by

$$
\begin{aligned}
I_{1-w_{1} ; w_{2}} \equiv & \int_{0}^{1} d w_{1} \int_{0}^{w_{1}} d w_{2} w_{1}^{2 \alpha^{\prime} k_{3} q_{1}}\left(1-w_{1}\right)^{2 \alpha^{\prime} k_{2} q_{1}-1} \\
& \times w_{2}^{2 \alpha^{\prime} k_{3} q_{2}-1}\left(1-w_{2}\right)^{2 \alpha^{\prime} k_{2} q_{2}}\left(w_{1}-w_{2}\right)^{2 \alpha^{\prime} q_{1} q_{2}} \\
= & \int_{0}^{1} d w_{1} w_{1}^{2 \alpha^{\prime}\left(k_{3}\left(q_{1}+q_{2}\right)+q_{1} q_{2}\right)}\left(1-w_{1}\right)^{2 \alpha^{\prime} k_{2} q_{1}-1} \\
& \times \int_{0}^{1} d t t^{2 \alpha^{\prime} k_{3} q_{2}-1}(1-t)^{2 \alpha^{\prime} q_{1} q_{2}}\left(1-t w_{1}\right)^{2 \alpha^{\prime} k_{2} q_{2}} \\
\sim & \frac{1}{\left(2 \alpha^{\prime} k_{2} q_{1}\right)\left(2 \alpha^{\prime} k_{3} q_{2}\right)}
\end{aligned}
$$

where we have kept only the first term (1) of the expansion of the last term in the previous equation. The last integral to compute is the one with the terms $w_{1}\left(1-w_{2}\right)$ in the denominator:

$$
\begin{aligned}
I_{w_{1} ; 1-w_{2}} \equiv & \int_{0}^{1} d w_{1} \int_{0}^{w_{1}} d w_{2}\left(1-w_{1}\right)^{2 \alpha^{\prime} q_{1} k_{2}} w_{1}^{2 \alpha^{\prime} q_{1} k_{3}-1}\left(w_{1}-w_{2}\right)^{2 \alpha^{\prime} q_{1} q_{2}}\left(1-w_{2}\right)^{2 \alpha^{\prime} q_{2} k_{2}-1} w_{2}^{2 \alpha^{\prime} q_{2} k_{3}} \\
= & \int_{0}^{1} d w_{1} \int_{0}^{w_{1}} d w_{2}\left(1-w_{1}\right)^{2 \alpha^{\prime} q_{1} k_{2}} w_{1}^{2 \alpha^{\prime} q_{1} k_{3}-1} \\
& \times\left(w_{1}-w_{2}\right)^{2 \alpha^{\prime} q_{1} q_{2}}\left(-\frac{1}{2 \alpha^{\prime} q_{2} k_{2}} \frac{\partial}{\partial w_{2}}\right)\left(1-w_{2}\right)^{2 \alpha^{\prime} q_{2} k_{2}} w_{2}^{2 \alpha^{\prime} q_{2} k_{3}} \\
= & \int_{0}^{1} d w_{1} \int_{0}^{w_{1}} d w_{2}\left(1-w_{1}\right)^{2 \alpha^{\prime} q_{1} k_{2}} w_{1}^{2 \alpha^{\prime} q_{1} k_{3}-1}\left(w_{1}-w_{2}\right)^{2 \alpha^{\prime} q_{1} q_{2}} \\
& \times\left(1-w_{2}\right)^{2 \alpha^{\prime} q_{2} k_{2}} w_{2}^{2 \alpha^{\prime} q_{2} k_{3}}\left[\frac{q_{2} \cdot k_{3}}{q_{2} k_{2}} w_{2}^{-1}-\frac{q_{1} q_{2}}{q_{2} k_{2}}\left(w_{1}-w_{2}\right)^{-1}\right]=0
\end{aligned}
$$

as one can see from eqs. (A.34) and (A.26). Finally, the term involving $I_{w_{1}-w_{2} ; w_{1}-w_{2}}$ is directly seen to be subleading in the double-soft limit, due to the explicit factor of $q_{1} q_{2}$ that multiplies this integral, and is thus not needed.

Open Access. This article is distributed under the terms of the Creative Commons Attribution License (CC-BY 4.0), which permits any use, distribution and reproduction in any medium, provided the original author(s) and source are credited.

\section{References}

[1] F.E. Low, Scattering of light of very low frequency by systems of spin $1 / 2$, Phys. Rev. 96 (1954) 1428 [inSPIRE].

[2] M. Gell-Mann and M.L. Goldberger, Scattering of low-energy photons by particles of spin 1/2, Phys. Rev. 96 (1954) 1433 [InSPIRE].

[3] S. Saito, Low-energy theorem for Compton scattering, Phys. Rev. 184 (1969) 1894 [INSPIRE].

[4] F.E. Low, Bremsstrahlung of very low-energy quanta in elementary particle collisions, Phys. Rev. 110 (1958) 974 [INSPIRE]. 
[5] S. Weinberg, Photons and gravitons in S-matrix theory: derivation of charge conservation and equality of gravitational and inertial mass, Phys. Rev. 135 (1964) B1049 [INSPIRE].

[6] S. Weinberg, Infrared photons and gravitons, Phys. Rev. 140 (1965) B516 [InSPIRE].

[7] T.H. Burnett and N.M. Kroll, Extension of the low soft photon theorem, Phys. Rev. Lett. 20 (1968) 86 [INSPIRE].

[8] J.S. Bell and R. Van Royen, On the low-burnett-kroll theorem for soft-photon emission, Nuovo Cim. A 60 (1969) 62 [inSPIRE].

[9] V. Del Duca, High-energy Bremsstrahlung theorems for soft photons, Nucl. Phys. B 345 (1990) 369 [inSPIRE].

[10] D.J. Gross and R. Jackiw, Low-energy theorem for graviton scattering, Phys. Rev. 166 (1968) 1287 [INSPIRE].

[11] R. Jackiw, Low-energy theorems for massless bosons: photons and gravitons, Phys. Rev. 168 (1968) 1623 [INSPIRE].

[12] V. de Alfaro, S. Fubini, G. Furlan and C. Rossetti, Currents in hadron physics, North-Holland, Amsterdam Netherlands (1974).

[13] E. Laenen, G. Stavenga and C.D. White, Path integral approach to eikonal and next-to-eikonal exponentiation, JHEP 03 (2009) 054 [arXiv: 0811.2067] [INSPIRE].

[14] E. Laenen, L. Magnea, G. Stavenga and C.D. White, Next-to-eikonal corrections to soft gluon radiation: a diagrammatic approach, JHEP 01 (2011) 141 [arXiv:1010.1860] [INSPIRE].

[15] C.D. White, Factorization properties of soft graviton amplitudes, JHEP 05 (2011) 060 [arXiv:1103.2981] [INSPIRE].

[16] A. Strominger, On BMS invariance of gravitational scattering, JHEP 07 (2014) 152 [arXiv:1312.2229] [INSPIRE].

[17] T. He, V. Lysov, P. Mitra and A. Strominger, BMS supertranslations and Weinberg's soft graviton theorem, JHEP 05 (2015) 151 [arXiv: 1401.7026] [INSPIRE].

[18] D. Kapec, V. Lysov, S. Pasterski and A. Strominger, Semiclassical Virasoro symmetry of the quantum gravity $\mathcal{S}$-matrix, JHEP 08 (2014) 058 [arXiv:1406.3312] [INSPIRE].

[19] H. Bondi, M.G.J. van der Burg and A.W.K. Metzner, Gravitational waves in general relativity. VII. Waves from axi-symmetric isolated systems, Proc. Roy. Soc. Lond. A 269 (1962) 21 [INSPIRE].

[20] R.K. Sachs, Gravitational waves in general relativity. VIII. Waves in asymptotically flat space-time, Proc. Roy. Soc. Lond. A 270 (1962) 103 [inSPIRE].

[21] G. Barnich and C. Troessaert, Symmetries of asymptotically flat four-dimensional spacetimes at null infinity revisited, Phys. Rev. Lett. 105 (2010) 111103 [arXiv:0909.2617] [INSPIRE].

[22] G. Barnich and C. Troessaert, BMS charge algebra, JHEP 12 (2011) 105 [arXiv:1106.0213] [INSPIRE].

[23] G. Barnich and C. Troessaert, Comments on holographic current algebras and asymptotically flat four dimensional spacetimes at null infinity, JHEP 11 (2013) 003 [arXiv:1309.0794] [INSPIRE].

[24] F. Cachazo and A. Strominger, Evidence for a new soft graviton theorem, arXiv:1404.4091 [INSPIRE]. 
[25] E. Casali, Soft sub-leading divergences in Yang-Mills amplitudes, JHEP 08 (2014) 077 [arXiv: 1404.5551] [INSPIRE].

[26] B.U.W. Schwab and A. Volovich, Subleading soft theorem in arbitrary dimensions from scattering equations, Phys. Rev. Lett. 113 (2014) 101601 [arXiv:1404.7749] [INSPIRE].

[27] N. Afkhami-Jeddi, Soft graviton theorem in arbitrary dimensions, arXiv:1405.3533 [INSPIRE].

[28] A.J. Larkoski, Conformal invariance of the subleading soft theorem in gauge theory, Phys. Rev. D 90 (2014) 087701 [arXiv: 1405.2346] [InSPIRE].

[29] M. Bianchi, S. He, Y.-t. Huang and C. Wen, More on soft theorems: trees, loops and strings, Phys. Rev. D 92 (2015) 065022 [arXiv:1406.5155] [InSPIRE].

[30] T. Adamo, E. Casali and D. Skinner, Perturbative gravity at null infinity, Class. Quant. Grav. 31 (2014) 225008 [arXiv:1405.5122] [INSPIRE].

[31] Y. Geyer, A.E. Lipstein and L. Mason, Ambitwistor strings at null infinity and (subleading) soft limits, Class. Quant. Grav. 32 (2015) 055003 [arXiv:1406.1462] [INSPIRE].

[32] H. Luo, P. Mastrolia and W.J. Torres Bobadilla, Subleading soft behavior of QCD amplitudes, Phys. Rev. D 91 (2015) 065018 [arXiv:1411.1669] [INSPIRE].

[33] J. Broedel, M. de Leeuw, J. Plefka and M. Rosso, Constraining subleading soft gluon and graviton theorems, Phys. Rev. D 90 (2014) 065024 [arXiv:1406.6574] [INSPIRE].

[34] Z. Bern, S. Davies, P. Di Vecchia and J. Nohle, Low-energy behavior of gluons and gravitons from gauge invariance, Phys. Rev. D 90 (2014) 084035 [arXiv:1406.6987] [INSPIRE].

[35] C. Kalousios and F. Rojas, Next to subleading soft-graviton theorem in arbitrary dimensions, JHEP 01 (2015) 107 [arXiv: 1407.5982] [INSPIRE].

[36] M. Zlotnikov, Sub-sub-leading soft-graviton theorem in arbitrary dimension, JHEP 10 (2014) 148 [arXiv:1407.5936] [INSPIRE].

[37] A.J. Larkoski, D. Neill and I.W. Stewart, Soft theorems from effective field theory, JHEP 06 (2015) 077 [arXiv: 1412.3108] [INSPIRE].

[38] W.-M. Chen, Y.-t. Huang and C. Wen, New fermionic soft theorems for supergravity amplitudes, Phys. Rev. Lett. 115 (2015) 021603 [arXiv:1412.1809] [INSPIRE].

[39] A. Sabio Vera and M.A. Vazquez-Mozo, The double copy structure of soft gravitons, JHEP 03 (2015) 070 [arXiv: 1412.3699] [INSPIRE].

[40] C.D. White, Diagrammatic insights into next-to-soft corrections, Phys. Lett. B 737 (2014) 216 [arXiv:1406.7184] [InSPIRE].

[41] Y.-J. Du, B. Feng, C.-H. Fu and Y. Wang, Note on soft graviton theorem by KLT relation, JHEP 11 (2014) 090 [arXiv:1408.4179] [INSPIRE].

[42] A.E. Lipstein, Soft theorems from conformal field theory, JHEP 06 (2015) 166 [arXiv: 1504.01364] [INSPIRE].

[43] E. Casali, Y. Geyer, L. Mason, R. Monteiro and K.A. Roehrig, New ambitwistor string theories, JHEP 11 (2015) 038 [arXiv:1506.08771] [INSPIRE].

[44] C. Cheung, K. Kampf, J. Novotny and J. Trnka, Effective field theories from soft limits of scattering amplitudes, Phys. Rev. Lett. 114 (2015) 221602 [arXiv:1412.4095] [INSPIRE]. 
[45] Z. Bern, S. Davies and J. Nohle, On loop corrections to subleading soft behavior of gluons and gravitons, Phys. Rev. D 90 (2014) 085015 [arXiv: 1405.1015] [INSPIRE].

[46] S. He, Y.-t. Huang and C. Wen, Loop corrections to soft theorems in gauge theories and gravity, JHEP 12 (2014) 115 [arXiv:1405.1410] [INSPIRE].

[47] J. Broedel, M. de Leeuw, J. Plefka and M. Rosso, Local contributions to factorized soft graviton theorems at loop level, Phys. Lett. B 746 (2015) 293 [arXiv:1411.2230] [INSPIRE].

[48] B.U.W. Schwab, Subleading soft factor for string disk amplitudes, JHEP 08 (2014) 062 [arXiv: 1406.4172] [INSPIRE].

[49] B.U.W. Schwab, A note on soft factors for closed string scattering, JHEP 03 (2015) 140 [arXiv: 1411.6661] [INSPIRE].

[50] P. Di Vecchia, R. Marotta and M. Mojaza, Soft theorem for the graviton, dilaton and the Kalb-Ramond field in the bosonic string, JHEP 05 (2015) 137 [arXiv: 1502.05258] [INSPIRE].

[51] M. Ademollo et al., Soft dilations and scale renormalization in dual theories, Nucl. Phys. B 94 (1975) 221 [inSPIRE].

[52] J.A. Shapiro, On the renormalization of dual models, Phys. Rev. D 11 (1975) 2937 [INSPIRE].

[53] T. Yoneya, String coupling constant and dilaton vacuum expectation value in string field theory, Phys. Lett. B 197 (1987) 76 [INSPIRE].

[54] H. Hata, Soft dilaton theorem in string field theory, Prog. Theor. Phys. 88 (1992) 1197 [INSPIRE].

[55] M. Bianchi and A.L. Guerrieri, On the soft limit of open string disk amplitudes with massive states, JHEP 09 (2015) 164 [arXiv: 1505.05854] [INSPIRE].

[56] N. Arkani-Hamed, F. Cachazo and J. Kaplan, What is the simplest quantum field theory?, JHEP 09 (2010) 016 [arXiv:0808.1446] [INSPIRE].

[57] I. Low, Adler's zero and effective Lagrangians for nonlinearly realized symmetry, Phys. Rev. D 91 (2015) 105017 [arXiv:1412.2145] [INSPIRE].

[58] F. Cachazo, S. He and E.Y. Yuan, New double soft emission theorems, Phys. Rev. D 92 (2015) 065030 [arXiv:1503.04816] [INSPIRE].

[59] F. Cachazo, S. He and E.Y. Yuan, Scattering of massless particles in arbitrary dimensions, Phys. Rev. Lett. 113 (2014) 171601 [arXiv:1307.2199] [INSPIRE].

[60] A. Volovich, C. Wen and M. Zlotnikov, Double soft theorems in gauge and string theories, JHEP 07 (2015) 095 [arXiv: 1504.05559] [INSPIRE].

[61] T. Klose, T. McLoughlin, D. Nandan, J. Plefka and G. Travaglini, Double-soft limits of gluons and gravitons, JHEP 07 (2015) 135 [arXiv: 1504.05558] [INSPIRE].

[62] G. Georgiou, Multi-soft theorems in gauge theory from MHV diagrams, JHEP 08 (2015) 128 [arXiv: 1505.08130] [INSPIRE]. 